\title{
Love in the Time of HIV: Testing as a Signal of Risk
}

\author{
Laura Derksen* \\ University of Toronto \\ Adamson Muula \\ Malawi College of Medicine \\ Joep van Oosterhout \\ Dignitas International
}

May 11, 2016

\begin{abstract}
The HIV epidemic in southern Africa has important consequences for economic development. The epidemic could be stopped by a universal test and treat policy, as antiretroviral drugs block the spread of the virus. However, demand for HIV testing and treatment are surprisingly low. This paper develops a model in which the decision to seek an HIV test is a signal of infection, and those who seek a test are subject to statistical discrimination from potential sexual partners. We evaluate an information experiment designed to test the theory, and find evidence that this form of discrimination is a significant barrier to HIV testing. In particular, we provide information at the community level on the public benefit of antiretroviral therapy: because the drugs prevent HIV transmission, a person who is tested and treated for HIV is a relatively safe sexual partner. This information reduces discrimination and increases HIV testing, with the strongest effects in communities where the new information becomes common knowledge. The results demonstrate that discrimination towards HIV positive individuals can be due to rational behavior by a misinformed public, and that providing new information can be an effective way to mitigate its effects.
\end{abstract}

JEL Codes: C70, D82, D83, I12, I15, I18

\footnotetext{
*laura.derksen@utoronto.ca

Laura Derksen is grateful to her supervisor, Oriana Bandiera and her advisor, Greg Fischer, for their continued guidance and encouragement. This paper also benefited from discussions with Marcella Alsan, Nava Ashraf, Victoria Baranov, Tim Besley, Patrick Blanchenay, Gharad Bryan, Jon De Quidt, Erika Deserranno, Kate Dovel, Thiemo Fetzer, Maitreesh Ghatak, Markus Goldstein, Willa Friedman, Marcos Vera Hernandez, Jason Kerwin, Alan Manning, Sam Marden, Steve Pischke, Munir Squires, Balazs Szentes, Susan Watkins, and Kelly Zhang, as well as seminar and conference participants at CSAE 2016, Dartmouth, IAS 2015, LSE, Mathematica, Melbourne, Ottawa, Oxford, PacDev 2016, PAA 2016, RES 2016, Sussex,
} 


\section{Introduction}

Over one million people in sub-Saharan Africa die of AIDS every year, despite the fact that an effective treatment, antiretroviral therapy (ART) is now widely provided to patients free of charge. The medication has large private benefits for those infected with HIV: it prolongs life by decades and reverses the symptoms of AIDS. It has recently been discovered that ART drugs also have a public benefit: they reduce HIV transmission by 96\% (Cohen et al., 2011). This discovery prompted the World Health Organization to advocate a "Treatment for Prevention" strategy to bring an end to the AIDS epidemic. Over the past decade, the supply of free ART has increased dramatically in low-income countries, yet demand for HIV testing and treatment remain low.

Various forms of discrimination towards people who seek HIV-related care are often blamed for low HIV testing rates. However, the underlying causes of discrimination are not well understood. Many policies designed to increase HIV testing afford clients more privacy, including door-to-door testing and self-testing. Other policies attempt to obscure the signal contained in a testing decision, for example, by offering monetary incentives to test (Thornton, 2008). Understanding the underlying desire for privacy is important in order to better predict the consequences of any policy, including the long-run impact, the population affected, and the effect on take-up of ART. Depending on the root causes of discrimination, it may also be possible to design policies which actually reduce the level of discrimination towards people living with HIV, rather than simply helping people to cope with it.

In this paper, we use a carefully designed information experiment to both detect and lower one specific barrier to HIV testing: statistical discrimination by potential sexual partners, based on a rational fear of HIV transmission. Those who seek an HIV test are more likely to be infected, and may face rejection from potential partners who fear contracting the virus. The public benefit of ART drugs implies that, in fact, an HIV test should not be viewed as a negative signal, since those who have been tested and treated for HIV are less contagious. We find that providing precise information, at the community level, on the public benefit of ART greatly increases the HIV testing rate within only four months, and we identify a reduction in discrimination between sexual partners as the likely mechanism. These results suggest that statistical discrimination between sexual partners who fear HIV transmission is a major barrier to HIV testing, and that correcting misconceptions about HIV transmission can mitigate discrimination and its effects.

In order to make precise the social interactions we have in mind, we model HIV testing as a signalling game between two potential sexual partners. One person decides whether to get tested for HIV, either at a clinic near his home village, or far away. He is then matched with a potential sexual partner. She observes his testing decision with some probability, which depends on his choice of testing location. She forms consistent beliefs about the risk of contracting HIV, and decides whether to reject or consent to the match.

Those who are infected with HIV have more to gain from HIV testing, because it

Toronto, UBC, and UWO. We gratefully acknowledge assistance with field work and data collection from the Malawi College of Medicine, Dignitas International and Invest in Knowledge Initiative, as well as financial support from LSE, STICERD and the Russell Sage Foundation. All errors remain those of the authors. 
allows them to access ART drugs. This causes a partially separating equilibrium to emerge, in which some fraction of the population reject matches who have been tested, because they fear contracting HIV. This behavior results in statistical discrimination towards those who choose to test. An upward shift in beliefs about the public benefit of ART alleviates this particular form of discrimination, and leaves other potential forms of social stigma unaffected. That is, people should now be more likely to consent to matches who have been tested and treated, because they know that treatment prevents HIV transmission. This should, in turn, increase HIV testing.

By mapping the model to an information experiment, we are able to test several theoretical predictions empirically. We randomly assigned 122 villages in Malawi to either partial or full intervention arms, and another 122 to a pure control arm. In all intervention villages we organized a campaign in which information about ART was disseminated in public, at community health meetings. In full intervention villages, information on both the private and public benefits of ART was provided, in particular, meeting attendees were informed that ART reduces HIV transmission by $96 \%$. In the partial intervention we provided information on only the private benefits and availability of ART.

While the formation of beliefs may in general depend on endogenous factors such as education and past health seeking behavior, a randomized experiment allows us to measure the causal effect of beliefs about the benefits of ART drugs. We are also interested in evaluating the information intervention itself, for its potential to inform policy. Without an information campaign, incorrect beliefs might persist in equilibrium. First, ART is under-adopted, which means that first-hand learning is slow. Second, information sharing between social contacts may be strategic and lack credibility; knowledge about ART may be viewed as a signal of HIV status, and a potential sexual partner who claims that ART blocks HIV transmission may have ulterior motives.

Our empirical results are consistent with the theoretical predictions of the signalling model. Survey measures of discrimination towards potential sexual partners who are HIV positive, but treated with ART, were significantly lower in the full intervention group than in the partial intervention group. Health seeking behavior also increased significantly. The HIV testing rate, as recorded over four months of administrative data, was $27 \%$ higher in full intervention villages than in partial intervention villages. Overall, nearly half of HIV tests take place far away from the HIV testing client's home village, but a large shift in beliefs about the public benefit of ART predicts an increase only in the number of tests sought nearby. The individual testing decision is predicted by common knowledge within the community about the public benefit of ART, and not by one's own beliefs; if the results were driven by altruism, one would expect the opposite.

Both the partial and full interventions caused a significant increase in uptake of ART drugs among those who had already tested positive. This demonstrates that community outreach is an effective strategy to increase demand for treatment and prevent new infections. This also suggests that some HIV positive individuals were unaware of the private benefits or availability of ART drugs, and that providing accurate, up-to-date information should be part of any community outreach policy to increase demand for HIV testing and treatment.

This paper is a study on the cause and effect of statistical discrimination, and its lessons may apply broadly. We show that a technology may be underadopted 
if it primarily benefits a group that is subject to statistical discrimination, and that correcting wrong beliefs may diminish discrimination and its effects. Arrow (1973)'s seminal work on statistical discrimination shows that a correlation between individual attributes and productivity results in labor market discrimination. The theoretical and empirical effects of this type of discrimination have been investigated in various contexts $^{1}$. Much of the economic literature on social stigma, both in the context of HIV and more generally, views stigma as an exogenous cost ${ }^{2}$, and investigates the effects of such a cost. Benabou and Tirole (2011) and Besley and Coate (1992) provide theoretical explanations for stigma, either as a social tool to discourage undesirable behavior or statistical discrimination, and investigate the theoretical implications of such models. In this paper we investigate both the source and effect of stigma, theoretically and empirically, by microfounding stigma as statistical discrimination against HIV-infected sexual partners, and using an experiment to investigate the implications for health seeking behavior.

A lack of accurate information may explain the low adoption rates of many health measures. Providing new, precise information on health risks has been shown to impact some types of behavior ${ }^{3}$. Within the context of HIV, most papers focus on the effect of information ${ }^{4}$ on risk-taking (Bandiera et al., 2012, Dupas, 2011, Kerwin, 2014). However, the type of information which might increase health seeking behavior is not well understood. Information on the protective benefits of circumcision appears to have no effect on demand for the procedure (Chinkhumba et al., 2012, Godlonton et al., 2011). Health seeking behavior may often be viewed as a signal of illness or infection, and our paper suggests that providing information specifically on the public benefit of health seeking behavior may effectively reduce the level of discrimination towards those who seek care.

Previous findings by Thornton (2008) show that test-seeking responds strongly to small monetary incentives, which conceal a person's true motivation for testing, and Ngatia (2011) shows that the decision to respond to such incentives carries a reputational cost. These experiments also offer effective policy advice in terms of HIV testing. However, providing monetary incentives for ART take-up may prove infeasible. In order to obscure the signal, the entire community, and not only ART patients, would have to be paid to visit the clinic at regular intervals. An intervention that instead reduces the level of discrimination towards people who seek HIV-related care is attractive for several reasons. Such an intervention should increase both HIV testing and treatment, and generate persistent effects for a fixed cost. In addition, we show that discrimination is likely to be practiced between potential sexual partners, which has important implications for policy. Discrimination imposes a high cost on those individuals most concerned with their sexual prospects, the same individuals who are most at risk of HIV infection. Designing policy to increase HIV testing and treatment

\footnotetext{
${ }^{1}$ See for example, Altonji and Pierret (2001), and Coate and Loury (1993).

${ }^{2}$ In the context of HIV, see Ngatia (2011) and Thornton (2008), and in the context of welfare payments, and Moffitt (1983).

${ }^{3}$ See, for example Bandiera et al. (2012), Dupas (2011), Jalan and Somanathan (2008), Kerwin (2014), and Madajewicz et al. (2007).

${ }^{4}$ Interventions which provide new, precise information differ sharply from traditional anti-HIV messaging strategies which focus on behavior change directly. Duflo et al. (2012), for example, find that a behavior change program that pushes abstinence fails to reduce risk-taking among adolescent girls in Kenya.
} 
among this group should be a priority.

The remainder of the paper proceeds as follows: Section 2 describes the experimental context and design, Section 3 presents the model and its predictions, Section 4 provides a description of the data and results, and Section 5 concludes.

\section{Background and Experimental Design}

\subsection{Antiretroviral Therapy}

Antiretroviral therapy, or ART, is a combination of drugs which suppress the HIV virus and reverse the progression of AIDS. The medication dramatically reduces the mortality and morbidity associated with HIV infection.

ART was developed in the late 1980's. However, the price of ART was prohibitively high for the low-income countries most affected by the AIDS epidemic. Around 2000, multiple agreements between governments, international organizations, and pharmaceutical companies led to a large reduction in the price of ART in developing countries. Over the next decade, HIV patients in Africa receiving ART drugs increased from 50,000 to 9.7 million, resulting in an approximate $30 \%$ decrease in mortality (WHO, 2013).

While the increased supply of ART has led to a remarkable improvement in outcomes for those infected with HIV, demand for the medication is surprisingly low. In sub-Saharan Africa, half of the population has never been tested for HIV, and only one third of those infected are taking ART drugs (WHO, 2013). There are more than one million AIDS-related deaths in the region every year, most of which could be prevented by ART (WHO, 2013).

In addition to providing large health benefits to HIV positive individuals, ART reduces HIV transmission between sexual partners by suppressing an infected person's viral load. A large randomized controlled trial ${ }^{5}$ recently demonstrated that regular ART use by HIV positive individuals reduces HIV transmission by $96 \%$ (Cohen et al., 2011). The World Health Organization and others have called for a "treatment for prevention" strategy, advocating ART use specifically for the purpose of preventing new infections. Epidemiological models suggest that in light of the public benefit of ART, universal testing and treatment could bring an end to the AIDS epidemic within 50 years, and would be cost-effective in the long run (Granich et al., 2009).

\subsection{Discrimination and HIV}

Because HIV is transmitted through sexual interactions, it is particularly susceptible to many forms of discrimination and social stigma ${ }^{6}$, including various forms of statistical discrimination, a distaste for social interactions with individuals having a particular

\footnotetext{
${ }^{5}$ This randomized trial was conducted in the field and in low-income countries. The drug reduced HIV transmission by $96 \%$, measured over a period of four years, among couples who took the drug at home, unobserved, and in general in a similar manner to most ART patients in the developing world. In particular, this reduction in transmission does not hinge on perfect adherence to the medication. It is accurate to say that ART drugs reduce HIV transmission by at least $96 \%$ relative to the absolute transmission rate over any time period up to approximately ten years. The absolute transmission rate is low, so the the total reduction in transmission over repeated interactions is approximately linear.

${ }^{6}$ See Mahajan et al. (2008) for a review of medical and sociological research on the stigmatization of HIV and AIDS.
} 
attribute, or an equilibrium of social behavior that arises spontaneously (Peski and Szentes, 2013) or that discourages undesirable behavior (Benabou and Tirole, 2011). Hoffmann et al. (2014) find that respondents in rural Kenya are willing to forgo payment to avoid coming into contact with an object handled by an HIV positive person. This may be because individuals may wish to disassociate themselves from others who have engaged in sexual behavior that is deemed socially unacceptable (Ngatia, 2011). Discrimination may also be purely statistical: a person might reject sexual partners who are HIV positive out of a rational fear of infidelity or HIV transmission.

While HIV positive individuals may face discrimination from many sources including employers, family members and social contacts, discrimination from potential sexual partners may be particularly common, due to the fact that HIV is sexually transmitted and many fear contracting the virus. Discrimination from potential partners may also be costly for many Malawians, even those who are married or in committed relationships. Helleringer et al. (2009) find that multiple concurrent sexual relationships are common in Malawi, and strongly predict HIV infection. In addition, cash transfers to young women in Malawi were shown to reduce both sexual behavior (Baird et al., 2010) and HIV infection (Baird et al., 2012), which suggests that new sexual partners are a source of financial support for many young women.

Discrimination towards people living with HIV may explain why testing levels are low across sub-Saharan Africa, and in Malawi, where our study takes place. The correlation between HIV status and HIV testing implies that any negative reaction towards those who are HIV positive may also apply to test seekers.

In order to obtain ART drugs, a person must first seek an HIV test. While the results of an HIV test are private ${ }^{7}$, the decision to test may be observable. The availability of ART drugs induces adverse selection among test seekers; for example, in Malawi, true HIV prevalence is $44 \%$ higher among those who report having been tested (DHS, 2010). The fact that seeking an HIV test is associated with HIV infection may generate stigma, which in turn discourages HIV testing; in Malawi, 27\% of women and $48 \%$ of men report never having been tested for HIV (DHS, 2010). In addition, our data shows that $49 \%$ of HIV tests do not take place at a nearby health facility, which could be explained by a desire to avoid being seen.

The Malawian Journals Project ${ }^{8}$ provides extensive qualitative evidence on social interactions surrounding HIV-related care. The project has transcribed a vast amount of hearsay data on attitudes towards HIV, and these journals include many examples of negative reactions towards HIV testing and treatment, especially in the context of the dating market. Many express dismay at the widespread availability of ART, as the medication makes HIV positive individuals appear healthy and therefore harder to identify. Others report seeking HIV testing and treatment far from home, to avoid being seen and then rejected by potential sexual partners. Using qualitative data from these journals, Angotti et al. (2016) find that while the private benefits of ART are well understood, the benefits to the community are not: community acceptability of ART use is low, and ART use is believed to be linked with an increased risk of HIV transmission.

\footnotetext{
${ }^{7}$ In Malawi, non-joint HIV tests are confidential, and a person who tests HIV negative does not receive any written proof of the result. The test is conducted twice with different test kits, so an incorrect diagnosis is unlikely.

${ }^{8}$ http:/ / malawi.pop.upenn.edu/malawi-data-qualitative-journals
} 


\subsection{Setting and Randomization}

This paper evaluates an experiment which took place in Zomba District, Malawi. Malawi is representative of Southern Africa in terms of its relatively high rate of HIV infection, and recent but widespread access to ART drugs. In Zomba District, HIV prevalence is approximately $14.5 \%$ (DHS, 2010), and both HIV testing and antiretroviral therapy (ART) are available for free at rural clinics.

The study covers two areas, TA Chikowi and TA Mwambo (see Figure 1), within Zomba District. We include only villages with unique names in the district, which enables us to identify the study villages in administrative clinic data. From this set, we selected a random sample of 244 villages; 122 were assigned to the control group, and the other 122 were randomly assigned to either partial or full intervention arms. We stratified on population and nearest health facility. The sample appears balanced on observable village characteristics (see Table 2).

The average village population is 440 , with approximately half between the ages of 15 and 49. All villages are within 11 kilometers of a health facility, and the average distance is 5 kilometers. Many villages are very close to one another; $60 \%$ have another study village within 1 kilometer. For this reason, accounting for spillovers will be an important part of the analysis in Section 4.

\subsection{Intervention}

An information intervention took place in each of the 122 intervention villages, in the form of a community health education meeting sanctioned by the Malawi College of Medicine and the village chief, and conducted by ten community educators ${ }^{9}$. The target population consisted of men and women aged 15 to 49 .

We conducted both partial and full information interventions in order to isolate the effect of one particular piece of information: the public benefit of ART drugs, that is, their capacity to prevent HIV transmission. In the partial intervention we provided information on the private benefits of ART drugs, while in the full intervention we provided information on both the private and public benefits of ART. In this way, we avoid conflating the effect of beliefs about the public benefit of ART with the effect of information on the existence, private benefit, or availability of ART drugs.

In partial intervention villages, community educators started the meeting by eliciting a rough measure of initial beliefs: a show of hands to determine whether meeting attendees were aware of the private benefits of ART. $75 \%$ of attendees believed that ART allowed HIV positive individuals to lead a long and healthy life. Educators then informed meeting attendees that ART increases life expectancy, hides the symptoms of AIDS, and is free at local clinics. They emphasized the importance of correct adherence, and that condom use and abstinence were good methods of HIV prevention. They did not discuss the link between ART use and HIV transmission. Educators used an infographic depicting a reduction in viral load to explain how ART alleviates the symptoms of AIDS (Figure 3).

In full intervention villages, community educators provided information on both the private and public benefits of ART drugs. First, they provided the same basic infor-

\footnotetext{
${ }^{9}$ These meetings took place in November and December, 2013. The educators had official identification badges from the Malawi College of Medicine which were meant to increase their credibility.
} 
mation they provided in the partial intervention. Next, they elicited a measure of initial beliefs about the public benefit of ART: a show of hands indicated that the public benefit of ART was initially unknown to most community members; on average, only $5 \%$ of meeting attendees believed that ART drugs had any effect at all on HIV transmission. Educators then explained the magnitude of the public benefit: that ART reduces the probability of HIV transmission by $96 \%$. They also used an infographic depicting not only the fact that ART reduces a person's viral load, but that this reduction in viral load leads to a reduction in transmission risk (Figure 4). The community educators emphasized that this reduction in HIV risk was based on correct adherence to ART as prescribed.

A detailed description of the information provided in all intervention villages is shown in Table 1. Educators followed a carefully worded script, and answered questions only by repeating information already provided. Unanswered questions were referred to the nearest health facility. The meetings were balanced in terms of length (approximately 45 minutes) and community participation; the partial and full interventions involved different show-of-hands questions and infographics. The information content of the meetings was identical, except for the critical discussion about HIV transmission.

The community educators first conducted meetings in the partial intervention villages, which took place over ten days. At the end of these ten days, training on the full intervention took place, and meetings took place over the following ten days. We conducted the two interventions sequentially in order to prevent information spillovers due to the conduct of the community educators. The team of educators traveled together to a different geographical section of Zomba District on each day, and within that area they were randomly assigned to different villages. Small villages were assigned one educator, while in villages with over 100 households work was done in pairs. Educators disseminated information through a combination of the meeting organized by the village chief and door-to-door campaigning. The educators knew they were to receive large incentives based on knowledge retention in the specific partial and full intervention villages they had visited, according to data from a survey five months after the intervention ${ }^{10}$. While educators knew the maximum size and timing of the incentive, the exact relationship between the survey questions and the size of the incentive were left intentionally undefined in order to avert any attempt to "teach to the test."

Attendance levels at community health meetings were high. An average of $67 \%$ of the target population was reached in each village, and approximately two thirds of attendees were women. It does not appear as though the community educators learned to attract more meeting attendees over time; the average attendance level was insignificantly lower in the full intervention group (65\%) than in the partial intervention group (68\%). The topic of the meeting was not announced ahead of time, so the sample of meeting attendees should be similar in all villages. Village chiefs received small gifts of soap and salt for organizing and advertising the meetings, but attendees did not

\footnotetext{
${ }^{10}$ The maximum incentive was approximately 100 USD. These incentives were paid to community educators in May, 2014, and the size of the incentive was based on the percent of community meeting attendees in full intervention villages who believed that ART prevents HIV transmisson, and the percent of community meeting attendees in partial intervention villages who believed that ART had private benefits, as recorded in the survey.
} 
receive any incentive for attendance.

\section{Model of HIV Testing as a Signal}

The aim of this section is to provide a signalling model of the HIV testing decision, and to illustrate a clean empirical test of the theory. This empirical test motivates the information experiment described in Section 2.4.

The idea that the choice to test for HIV provides and observable and informative signal is related in some ways to the sociological concept of stigma. We define HIVrelated stigma as a phenomenon whereby individuals who are suspected of having HIV are excluded from social interactions ${ }^{11}$. This paper focuses on one potential source of stigma, and one particular type of social interaction. Individuals who are likely to be HIV positive may be excluded from sexual interactions due to a rational fear of contagion. If HIV testing is a signal of underlying risk, that is, high-risk individuals are more likely to seek an HIV test, then those who do so may face statistical discrimination by potential sexual partners. Other types of stigma, and other barriers to HIV testing ${ }^{12}$ may exist, and are included in the model in reduced form ${ }^{13}$. Comparative statics will show that the information experiment reduces stigma if and only if it is due to a fear of HIV transmission; other forms of stigma and costs of testing remain unaffected.

\subsection{Environment}

We model the HIV testing decision as a one-sided signalling game between two players, over two periods. In the first period, the first player decides whether to seek an HIV test based on his private type, which corresponds to his risk of being HIV positive. In the second period, he is matched at random to a potential sexual partner, who might observe whether or not he has been tested for HIV. Based on this signal, the second player decides whether or not to consent to a sexual relationship.

Consider a continuum of agents $\mathcal{A}=[0,1]$, and another continuum of equal measure $\mathcal{B}=[0,1]$ which represents potential sexual partners for agents in $\mathcal{A}$. A fraction $\bar{h}$ of individuals $a \in \mathcal{A}$ are HIV positive, while every $b \in \mathcal{B}$ is HIV negative ${ }^{14}$. We denote HIV status by $h_{a} \in\{0,1\}$.

We assume that individuals possess asymmetric information about their own HIV risk, as they have full knowledge of past risk-taking behavior and current symptoms. In the first period, each agent $a \in \mathcal{A}$ privately observes his type $\theta_{a}$. This represents his probability of being infected, which takes one of two values:

$$
\theta_{a} \in\left\{\theta_{L}, \theta_{H}\right\}
$$

\footnotetext{
${ }^{11}$ This is derived from the seminal definition by Goffman (1963), who defines stigma as "The phenomenon whereby an individual with an attribute which is deeply discredited by his/her society is rejected as a result of the attribute."

${ }^{12} \mathrm{HIV}$ testing has increased significantly over the past decade, in part due to increased supply of HIV testing services and ART. We do not wish to suggest that stigma is the only potential barrier to HIV testing.

${ }^{13}$ For ease of exposition, we include only stigma from potential sexual partners. Allowing for stigma from the general population does not produce a substantially different model or predictions.

${ }^{14}$ We shall refer to $a$ as "he" and $b$ as "she" for clarity, but the model is not meant to be gender specific. We use a one-sided signalling model for clarity of exposition; a two-sided model gives the same predictions.
} 
with $0<\theta_{L}<\theta_{H}<1$. We refer to these two types as low-risk and high-risk types, respectively.

The agent $a$ then decides whether or not to seek an HIV test, and if so, whether to test at a far away clinic $t_{a}^{f} \in\{0,1\}$ or nearby $t_{a}^{n} \in\{0,1\}$, such that $t_{a}^{n}+t_{a}^{f} \in\{0,1\}$ (agents cannot test in both locations). HIV testing is beneficial to those who are infected, as it provides immediate access to ART drugs ${ }^{15}$. The total payoff to HIV testing is $v$ if the test result is positive, and 0 otherwise. The parameter $v$ includes both the benefit of ART drugs, and other benefits such as counselling, social programs for people living with HIV, and easier access to other medication ${ }^{16}$.

The cost of traveling to a nearby clinic is $c^{n}$, while traveling to a far away clinic $\operatorname{costs} c^{f}$. These costs are not necessarily monetary: they include the opportunity cost of time, the probability that the clinic is closed, and the psychic cost of learning one's HIV status.

We assume that for low-risk types, the direct cost of HIV testing is higher than the expected benefit of access to medication, as the test result is unlikely to be positive. Meanwhile, for high-risk types, the expected benefit is higher than the direct cost.

$$
\theta_{L} v<c^{n}<\theta_{H} v
$$

We also assume that the cost of traveling to a far away clinic is bounded in the following way ${ }^{17}$.

$$
\frac{c^{f}-c^{n}}{1-\phi}<\theta_{H} v-c^{n}
$$

Agents $a \in \mathcal{A}$ receive the following payoff at the end of the first period.

$$
u_{1}^{a}\left(t_{a}^{n}, t_{a}^{f}\right)=t_{a}^{n}\left(h_{a} v-c^{n}\right)+t_{a}^{f}\left(h_{a} v-c^{f}\right)
$$

In the second period, each agent $a \in \mathcal{A}$ is matched at random with a potential sexual partner $b \in B$. While $b$ is aware of the population HIV prevalence $\bar{h}$, she does not observe $a^{\prime}$ s risk type directly, and she does not observe whether or not he is HIV positive.

Agent $b$ may, however observe $a$ 's testing decision. If $a$ tested nearby, he is observed with probability one, while if he tested far away, he is observed with probability $\phi \in$ $(0,1)$. This is modeled by a signal $\sigma_{a} \in\{0,1\}$ with $\mathbb{P}\left(\sigma_{a}=1\right)=t_{a}^{n}+\phi t_{a}^{f}$. Agent $b$ uses this signal to form beliefs about the probability that her partner $a$ is HIV positive; we denote these beliefs by $\hat{\theta}_{b}\left(\sigma_{a}\right)$.

Agent $b$ then decides whether to consent to a sexual relationship with her match, $m_{b} \in\{0,1\}$. If $b$ consents, $a$ and $b$ obtain relationship benefits $y_{a} \sim G$ and $y_{b} \sim G$ respectively ${ }^{18}$, where the distribution $G$ has positive support.

Consenting to the relationship comes with a cost to agent $b$; she risks becoming

\footnotetext{
${ }^{15}$ We assume that every person who tests positive starts ART drugs. This is equivalent to an assumption that the large benefits of ART drugs are known; we provide this information in the partial intervention described in 2.4.

${ }^{16}$ Many HIV positive patients require treatment for tuberculosis or sexually transmitted infections, as well as antibacterial medication which treats HIV-related infections.

${ }^{17}$ If this does not hold, then no equilibrium will exist in which agents test far away.

${ }^{18}$ We have assumed that $a$ always obtains net benefit from the relationship. We do not model his consent decision.
} 
infected with HIV. Because ART drugs reduce HIV transmission, the probability of contracting HIV from an infected partner depends on whether or not he has been tested and treated. The relative reduction in HIV transmission risk associated with ART use is denoted by $\rho$, and the true value is approximately 0.96 (Cohen et al., 2011). We denote the absolute transmission rate by $\tau$; this is the probability that an infected person transmits HIV to his sexual partner if he is not taking ART. The cost of contracting HIV is $c^{h}$.

We also allow for other forms of stigma. Agent $b$ may experience disutility from a relationship with an HIV-positive person distinct from the possibility of contracting HIV. This could be, for example, because of $a^{\prime}$ s propensity for infidelity, because of social norms, or because of a taste parameter. We capture all of these costs in reduced form, denoted by $c^{0}$.

If $b$ chooses to consent, the second-period payoff to agent $a$ is the benefit of the relationship.

$$
u_{2}^{a}\left(m_{b}\right)=m_{b} y_{a}
$$

The second-period payoff to agent $b$ depends on both the benefit of the relationship and the risk of contracting HIV.

$$
u_{2}^{b}\left(m_{b}\right)=m_{b}\left[y_{b}-h_{a}\left(c^{h} \tau\left(1-\rho\left(t_{a}^{n}+t_{a}^{f}\right)\right)+c^{o}\right)\right]
$$

In calculating the payoff to agent $b$ we make use of our assumption that everyone who seeks an HIV test receives ART immediately. This allows agent $b$ to conclude that an HIV-positive individual who has been tested is less contagious, as captured by the term $\left(1-\rho\left(t_{a}^{n}+t_{a}^{f}\right)\right)$.

We now make two additional assumptions. First,

$$
\mathbb{P}\left(y_{b}>\bar{h}\left(c^{h} \tau+c^{o}\right)\right)=1 ;
$$

the net payoff from a sexual relationship with the average, untreated member of the population is always positive. Second,

$$
\mathbb{P}\left(y_{b}<c^{h} \tau+c^{o}\right)=1 ;
$$

the net payoff from a sexual relationship with an untreated, HIV-positive match is always negative.

\section{Heterogeneous beliefs about the effect of ART}

In order to illustrate the link between the model and the experiment described in Section 2, we assume that agents differ in their perception of the risk of HIV transmission. Specifically, agents in $\mathcal{B}$ have heterogeneous beliefs about the extent to which ART drugs prevent the spread of HIV. Each agent $b \in \mathcal{B}$ has belief $\hat{\rho}_{b} \in[0,1]$ about the relative reduction in HIV transmission risk associated with ART. For example, $\hat{\rho}=0$ corresponds to the belief that ART has no effect on transmission, and $\hat{\rho}=1$ to the belief that it is impossible to contract HIV from a person taking ART.

We denote by $F_{\hat{\rho}}$ the distribution of beliefs ${ }^{19}$ in the population $\mathcal{B}$. We assume that

\footnotetext{
${ }^{19}$ We do not assume any relationship between the distribution of beliefs and the true value $\rho=0.96$.
} 
$F_{\hat{\rho}}$ is common knowledge among the population $\mathcal{A}$. This distribution of beliefs forms a key link between the model and our experimental design: our intervention provides information on the true value of $\rho$, which leads to a shift ${ }^{20}$ in the distribution $F_{\hat{\rho}}$.

\subsection{Strategies and Equilibria}

We solve for the pure-strategy Perfect Bayesian Equilibria of this game.

In the first period, agents in $\mathcal{A}$ choose an HIV-testing strategy $\left\{t^{n}, t^{f}\right\}$ to maximize their total expected utility ${ }^{21}$.

$$
\mathbb{E}\left(u_{1}^{a}+u_{2}^{a}\right),
$$

taking their private types, the strategies of $\{b \in \mathcal{B}\}$, as well as beliefs $F_{\hat{\rho}}$ and $\left\{\hat{\theta}_{b}(\sigma)\right.$ : $b \in \mathcal{B}\}$ as given.

In the second period, agents $b \in \mathcal{B}$ receive the signal $\sigma_{a}$ and form corresponding beliefs about $a^{\prime}$ s type $\hat{\theta}_{b}\left(\sigma_{a}\right)$, which are consistent on the equilibrium path. That is, agents use Bayes' rule to calculate the probability that a person is infected, taking into account whether or not they were observed seeking an HIV test. They then choose whether or not to consent in order to maximize their expected utility, based on their beliefs $\hat{\rho}_{b}$ and $\hat{\theta}_{b}$.

Proposition 1. There are two classes of pure-strategy Perfect Bayesian Equilibria in this game. The first is a partially separating equilibrium, in which a fraction $S$ of agents in $\mathcal{B}$ reject a match who has been observed seeking an HIV test, and the remaining fraction $1-S$ of agents consent to any match. The second is a pooling equilibrium, in which a fraction $P$ of agents in $\mathcal{B}$ consent if and only if their match has been observed seeking an HIV test.

We restrict attention to the partially separating equilibrium. The pooling equilibrium implies universal testing, which is not consistent with the low testing levels observed in the data. It is also unstable, in the sense that it does not satisfy the D1 criterion ${ }^{22}$ Discussion of this second equilibrium, as well as the proof that no other equilibria exist is deferred to Appendix A.

\section{The partially separating equilibrium}

We begin by characterizing the most important equilibrium for our purposes, in which there is discrimination towards those who seek HIV testing. In this equilibrium, every $b \in \mathcal{B}$ consents to a match who has not been observed testing, but a proportion $S$ of $\{b \in \mathcal{B}\}$ reject those who have been observed seeking an HIV test.

Given this strategy by agents in population $\mathcal{B}$, the decision to test for HIV is based on the expected impact on utility, which is equal to

$$
\theta_{a} v-c^{n}-S y_{a}
$$

Indeed, Figure 7 illustrates the extent to which beliefs are incorrect.

${ }^{20}$ The distribution of beliefs, as well as the effect of the intervention are illustrated in Figure 7.

${ }^{21}$ For simplicity and without loss of generality, we assume a discount rate of 1 .

${ }^{22}$ Intuitively, an equilibrium satisfies the D1 criterion if off-equilibrium deviators are assumed to have whatever risk type has a stronger incentive to undertake the deviation. In our setting, in an equilibrium with universal testing, a person who does not test should be perceived as a low-risk type. These offequilibrium beliefs would undermine the pooling equilibrium. 
if the test is sought at a nearby clinic, and

$$
\theta_{a} v-c^{f}-\phi S y_{a}
$$

if the test is sought at a far away clinic. In both cases, there is an indirect cost of testing due to the rejection agent $a$ might face from his sexual partner in the next period, and this cost is higher if $a$ chooses to test nearby.

By assumption (1), low-risk types will never seek an HIV test in this equilibrium. If $\theta_{a}=\theta_{H}, a$ will select an action as follows.

$$
\left(t_{a}^{n}, t_{a}^{f}\right)= \begin{cases}(0,0) & \text { if } \frac{T_{f}}{S}<y_{a} \\ (0,1) & \text { if } \frac{T_{n}}{S}<y_{a} \leq \frac{T_{f}}{S} \\ (1,0) & \text { if } y_{a} \leq \frac{T_{n}}{S}<\frac{T_{f}}{S}\end{cases}
$$

Where the thresholds are

$$
T_{f}=\frac{\theta_{H} v-c^{f}}{\phi}
$$

and

$$
T_{n}=\frac{c^{f}-c^{n}}{(1-\phi)}
$$

In the absence of discrimination or stigma from sexual partners $(S)$, all high-risk types would like to seek an HIV test nearby. However, if $S>0$, high-risk types will choose to not test, test far away, or test nearby depending on their benefit from a future relationship, $y_{a}$. Those who value a future sexual relationship very highly will not seek an HIV test. Agents with intermediate values if $y_{a}$ will choose far away testing, and those who value the relationship least will test nearby, as they have less to lose by being observed. In any case, the fact that only high-risk types seek HIV testing results in adverse selection.

We now turn our attention to the consent decision. If an agent $b$ does not observe her match seeking an HIV test $\left(\sigma_{a}=0\right)$, she will consent to a sexual relationship. This stems from assumption (6), which states that any agent $b \in \mathcal{B}$ would obtain net benefit from a relationship with the average member of the population $\mathcal{A}$. Because low-risk types do not test for HIV, they generate the signal $\sigma=0$; meanwhile, some high-risk types generate $\sigma=0$ and others generate $\sigma=1$. Therefore, the person $b$ has been matched with cannot be more likely to have HIV than the average member of the population. Formally, maximizing her expected payoff (5), $b$ will consent if

$$
y_{b}>\mathbb{E}\left[h_{a}\left(c^{h} \tau\left(1-\hat{\rho}_{b}\left(t_{a}^{n}+t_{a}^{f}\right)\right)+c^{o}\right) \mid \sigma_{a}=0\right]
$$


where we have replaced $\rho$ by the belief $\hat{\rho}_{b}$. The right-hand side of this inequality is always ${ }^{23}$ less than or equal to $\bar{h}\left(c^{h} \tau+c^{o}\right)$, so by assumption (6), $b$ will consent.

If, on the other hand, $b$ observes that $a$ has tested for HIV $\left(\sigma_{a}=1\right)$, she infers that he is a high-risk type. She will therefore consent if ${ }^{24}$

$$
y_{b} \geq \theta_{H}\left(c^{h} \tau\left(1-\hat{\rho}_{b}\right)+c^{o}\right) .
$$

Note that she will only consent if her benefit from the relationship $\left(y_{b}\right)$ is sufficiently high, or if she believes that ART is effective at preventing HIV transmission ( $\hat{\rho}_{b}$ is high).

We are now able to provide a formula for the total level of discrimination in the population, $S$, which we previously defined as the fraction of agents in $\mathcal{B}$ who would withhold consent from a match they have observed seeking an HIV test.

$$
S=\int_{0}^{\infty}\left(\int_{0}^{1+\left(c^{o} / c^{h} \tau\right)-\left(y / \theta_{H} c^{h} \tau\right)} f(\hat{\rho}) d \hat{\rho}\right) g(y) d y
$$

The functions $f$ and $g$ represent probability densities for $\hat{\rho}$ and $y$ respectively.

\subsection{Comparative Statics}

This paper evaluates an experiment that was designed to shift beliefs about the ART prevention parameter $\rho$, by disseminating the information that ART reduces HIV transmission by $96 \%$. In our model, we interpret this as a first-order stochastically dominant shift in the distribution $F_{\hat{\rho}}$. Indeed, we observe empirically that beliefs are biased towards 0 (most are unaware of the preventative effect of ART), and the information intervention appears to have shifted the distribution uniformly towards 1 (see Figure 7).

We now characterize the theoretical implications of such a shift in the distribution of beliefs.

Proposition 2. If $c^{h}=0$, a change in the distribution of beliefs $F_{\hat{\rho}}$ has no effect on the level of discrimination between sexual partners $S$.

This comes from (10): if $c^{h}=0$, a change in $\hat{\rho}_{b}$ has no effect on whether $b$ consents or not. Intuitively, if there is social stigma is based on something other than the risk of HIV transmission, then changing beliefs about the risk of transmission will not affect the level of stigma.

In what follows, we consider the case $c^{h}>0$, that is, there is some cost associated with contracting HIV.

Proposition 3. If $c^{h}>0$, a first-order stochastically dominant shift in the distribution of beliefs $F_{\hat{\rho}}$ results in a weak decrease in the level of discrimination between sexual partners $S$.

If the distribution of beliefs shifts upward, each agent is more likely to believe that ART is effective in reducing HIV transmission. For some of these agents, this will

\footnotetext{
${ }^{23}$ Proof: $\mathbb{E}\left(h_{a}\left(c^{h} \tau\left(1-\hat{\rho}_{b}\left(t_{a}^{n}+t_{a}^{f}\right)\right)+c^{o}\right) \mid \sigma_{a}=0\right) \leq \mathbb{E}\left(h_{a}\left(c^{h} \tau+c^{o}\right) \mid \sigma_{a}=0\right)=\mathbb{E}\left(\theta_{a} \mid \sigma_{a}=0\right)\left(c^{h} \tau+\right.$ $\left.c^{o}\right) \leq \mathbb{E}\left(\theta_{a}\right)\left(c^{h} \tau+c^{o}\right)=\bar{h}\left(c^{h} \tau+c^{o}\right)$

${ }^{24}$ Here, we make use of the fact that she is certain her match is a high-risk type, and has been tested for HIV: $t_{a}^{n}+t_{a}^{f}=1$. In this case, $\mathbb{E}\left[h_{a}\left(c^{h} \tau\left(1-\hat{\rho}_{b}\left(t_{a}^{h}+t_{a}^{f}\right)\right)+c^{o}\right) \mid \sigma_{a}=1\right]=\theta_{H}\left(c^{h} \tau\left(1-\hat{\rho}_{b}\right)+c^{o}\right)$.
} 
imply a change in strategy from not consenting to consenting. In aggregate, the level of discrimination will decrease. Formally, the level of discrimination can be written as

$$
S=\int_{0}^{\infty} F_{\hat{\rho}}\left(1+\frac{c^{o}}{s \tau}-\frac{y}{\theta_{H} c^{h} \tau}\right) g(y) d y .
$$

Consider a first-order stochastically dominant shift in the distribution $F_{\hat{\rho}}$. For each value of $y$, the integrand will decrease. Therefore, $S$ will also decrease. It is worth pointing out that if other forms of stigma $c^{0}$ are large relative to the cost of contracting $\mathrm{HIV}, c^{h}$, a shift in beliefs about $\rho$ will have no effect, as the integrand in (12) will equal 1 for any distribution.

Proposition 3 demonstrates that, by shifting beliefs, the direct effect of the information experiment is to reduce the level of discrimination between sexual partners at the community level, if that discrimination is based on a fear of contracting HIV. We next discuss the implications of this reduction in discrimination on HIV testing.

Proposition 4. A decrease in discrimination between sexual partners leads to an increase in the total number of HIV tests, and an increase in the number of tests at nearby clinics.

These two statements follow from the fact that a cumulative distribution function $G(y)$ always increases in $y$, and $\lim _{y \rightarrow \infty} G(y)=1$.

Proposition 5. A moderate decrease in S has an ambiguous effect on the number of HIV tests at far away clinics, but as $S \rightarrow 0$, the number of far away tests approaches zero.

The fraction of $\{a \in \mathcal{A}\}$ who choose far away testing equals

$$
G\left(\frac{\theta_{H} v-c^{f}}{\phi S}\right)-G\left(\frac{c^{f}-c^{n}}{(1-\phi) S}\right)
$$

which, depending on the distribution $G$, may be locally increasing or decreasing in $S$.

Figure 2 depicts the relationship between discrimination, testing levels, and testing location in the case where the distribution $G$ is normally distributed and initial testing levels are low. In this case, a moderate increase in beliefs results in an increase in far away testing, but as stated in Proposition 5, this is not a general result. The effect depends on the distribution $G$ and on initial testing levels.

The predicted change in HIV testing behavior comes from a decrease in discrimination, which is driven entirely by the beliefs of potential sexual partners. This leads to another specific empirical implication.

Proposition 6. The decision to test for HIV depends on the beliefs of the community $F_{\hat{\rho}}$, and not on one's own beliefs about the preventative nature of ART drugs.

The effect of a shift in beliefs on sexual behavior is not immediately clear. If discrimination against those who've been tested decreases, this may cause a direct increase in sexual behavior. However, the rate of HIV testing also increases, which increases the number of individuals subjected to discrimination from potential partners.

Proposition 7. The effect of the level of discrimination, $S$, on the number of high-risk individuals who obtain consent from their matches is ambiguous. 
Recall that all low-risk individuals $\left(\theta_{a}=\theta_{L}\right)$ do not test, and therefore obtain consent. A high-risk person $a$ with $\theta_{a}=\theta_{H}$ will be refused if he seeks a test, is observed by the community, and meets a discriminatory match. The fraction of high-risk types who obtain consent is equal to

$$
M\left(\theta_{H}\right)=1-\phi S G\left(T_{f} / S\right)-(1-\phi) S G\left(T_{n} / S\right) .
$$

If we take the first order condition with respect to the level of discrimination $S$, we obtain

$$
\frac{d M\left(\theta_{H}\right)}{d S}=\phi\left[g\left(\frac{T_{f}}{S}\right) \frac{T_{f}}{S}-G\left(\frac{T_{f}}{S}\right)\right]+(1-\phi)\left[g\left(\frac{T_{n}}{S}\right) \frac{T_{n}}{S}-G\left(\frac{T_{n}}{S}\right)\right]
$$

The sign of this derivative depends on the distribution of preferences $G$. For example, if $G$ is the Uniform distribution, the net effect of a decrease in discrimination on sexual behavior is zero.

\section{Empirical Strategy and Results}

We next turn our attention to the empirical results of the experiment described in Section 2. In order to test the assumptions of our model, we investigate the effect of the experiment on beliefs about ART transmission. We then test the main predictions of our model by examining the effect of the experiment on attitudes toward ART drugs, health seeking behavior, and sexual risk taking. Finally, we attempt to rule out two alternative mechanisms as first order explanations for the patterns of health seeking behavior we observe.

\subsection{The Effect of the Information Intervention on Beliefs}

As a first step, we ascertain whether the full information intervention achieved its goal of shifting beliefs about the public benefit of ART, and whether other types of beliefs were affected. We also investigate the effect of the full intervention on survey measures of discrimination between sexual partners.

In order to test the theory, and isolate the effect of a change in beliefs about the public benefit of ART, we must investigate the effect of the full intervention on beliefs and attitudes relative to the partial intervention.

\section{Survey data}

We conducted a survey in both partial and full intervention villages approximately four months after the intervention. Control villages were not surveyed. A total of 1,358 individuals were interviewed. The purpose of this survey was to collect individual data on beliefs, attitudes towards HIV, health seeking behavior, and sexual behavior.

The interviewers selected respondents by conducting a random walk within each village $^{25}$. The survey was administered to those who attended the community health

\footnotetext{
${ }^{25}$ Two interviewers were assigned to each village; one began the random walk at the center and the other at an outer edge of the village.
} 
meeting ${ }^{26}$. We also conducted a very brief survey among 536 non-attendees, in order to assess the spread of information within the community.

The interviewers were hired from a pool of candidates who were not socially connected to the community educators employed for the intervention. Interviewers had no information on the purpose of the original intervention, nor the identities of the treatment and control villages. Out of 122 study villages, 119 were successfully surveyed. In one full intervention village and two partial intervention villages, village authorities denied permission for a survey to take place due to the recent death of a village leader.

\section{Regression equation}

In order to investigate the effect of the full information intervention on the beliefs of meeting attendees, we regress each belief measure captured in the survey on the intervention arm of the village. Because the information intervention was randomly assigned, this allows us to estimate the causal effect of the information treatment on beliefs.

$$
\text { Belief }_{i j}=\alpha+\beta F_{j}+\delta^{\prime} \chi_{i j}+\epsilon_{i j}
$$

Here, Belie $f_{i j}$ is a belief measure elicited from respondent $i$ in village $j$ and $F_{j} \in$ $\{0,1\}$ is the indicator for whether village $j$ was assigned to the full intervention arm. The reference category is the partial intervention arm. $\chi_{i j}$ is a set of individual- and village-level covariates ${ }^{27}$. Standard errors are clustered at the village level. Because $F_{j}$ is randomly assigned, we expect $\mathbb{E}\left(\epsilon_{i j} \mid F_{j}\right)=0$, so the OLS estimate $\hat{\beta}$ is unbiased.

\section{Beliefs about the public benefit of ART increased}

Eliciting beliefs about the public benefit of ART was a primary goal of the survey, conducted approximately five months after the intervention. Five different measures were collected. First, respondents were presented with a list of possible HIV prevention methods, including the use of condoms, mosquito nets, circumcision, abstinence, and ART. They were asked to select those which reduce the probability of transmitting HIV. $80 \%$ of respondents in the full intervention group and only $19 \%$ in the partial intervention group chose ART as a prevention method (see Column 1 of Table 3).

Next, respondents were asked to agree or disagree (on a Likert five-point scale) with the following statment: ART reduces the probability that an HIV-positive person will spread the virus to his or her partner. The full information treatment had a significant effect; the average response in the partial intervention group was "disagree", while in the full intervention group the average response was "agree" (this measure is in Column 2 of Table 3, rescaled).

Respondents then ranked the following two types of potential sexual partners according to HIV transmission risk: a person who has never been tested for HIV, and a person who is HIV positive and taking ART correctly. Column 3 of Table 3 shows

\footnotetext{
${ }^{26}$ This results in selection bias among those interviewed relative to a random sample of the village as a whole, but this selection should be the same in both partial and full intervention villages.

${ }^{27}$ Individual-level controls include age, gender, whether the person is married, employed, primary school educated, secondary school educated, has livestock, and has a brick house. Village-level controls consist of covariates listed in Table 2 as well as village population and distance to each health facility.
} 
that in the partial intervention group, respondents said, on average, that a person who has never been tested for HIV is less risky, while in the full intervention group, most said that a person taking ART is a safer sexual partner. This suggests that many who attended the community meeting understood an important implication of the information provided: that a partner who has never been tested might in fact be more risky than a partner who is infected but treated.

In order to elicit beliefs about the rate of HIV transmission with and without ART drugs, we took inspiration from the bean-counting subjective beliefs measure advocated by Delevande and Kohler (2009). Respondents were shown ten bottle caps. Each bottlecap was meant to represent a serodiscordant couple: an HIV-positive person and his or her HIV-negative spouse. Respondents removed one bottle cap for each person who they believed would contract HIV within one year, assuming no one was taking ART. The process was then repeated, under the alternative assumption that all HIV-positive individuals took ART drugs as prescribed. Using these two measures of absolute and relative transmission rates, we calculate the beliefs about the relative reduction in risk associated with ART use. In Column 4 of Table 3, we see that based on this measure, individuals in the full intervention group believe that the relative reduction in HIV transmission risk is approximately $56 \%$; this is much higher than in the partial intervention group (9\%) but still well below the true value $\rho=0.96$.

We also capture beliefs about the ART prevention parameter $\rho$ using an infographic similar to the one displayed at the community health meetings (Figure 6). This measure was elicited last to ensure that respondents were not visually reminded of the information campaign before discussing their beliefs. Histograms in Figure 7 show that the distribution of beliefs about $\rho$ is uniformly higher in the full intervention group. In Column 5 of Table 3, we see that average beliefs $\hat{\rho}$ are significantly higher in the full intervention group, but below the true value $\rho=0.96$. It is reassuring that the measures in Columns 4 and 5 are somewhat similar; in the full intervention group, they correspond to beliefs $\hat{\rho}=0.56$ and $\hat{\rho}=0.70$ respectively.

When conducting brief surveys with those who did not attend a meeting, we elicited only the first two beliefs measures (Columns 1 and 2 of Table 3). There is some evidence of information spread from the meeting attendees to non-attendees, but only the second measure shows a significant effect.

\section{Beliefs about the private benefits of ART did not change}

Both partial and full intervention villages received basic information about the private benefits of ART drugs, and indeed, Column 3 of Table 4 shows that respondents' beliefs do not differ between the two intervention arms. Respondents were asked to agree or disagree, on a five-point Likert scale, with the statement An HIV-positive person can live a long and healthy life if he takes ART. This statement closely matches the intervention script. In all intervention villages, the average respondent strongly agreed with the statement.

\section{Beliefs about HIV transmission and prevalence did not change}

The full information campaign could have affected several beliefs about HIV. The knowledge that ART reduces HIV transmission may cause individuals to update their beliefs about the overall HIV prevalence in their community. In addition, while the absolute 
transmission rate was not mentioned during the information campaign, attendees may have formed some correct or incorrect beliefs about this rate based on the information that was provided. A shift in either of these beliefs may have an effect on HIV testing distinct from the effects predicted by the model described in Section 3. However, Table 4 shows that there were no significant changes in these beliefs.

As mentioned above, we elicited beliefs about the absolute transmission rate using ten bottle caps. We asked respondents how many HIV-negative individuals with infected, untreated partners would contract the virus over the course of one year. The respondent removed one bottle cap for each case of HIV transmission. We also used bottle caps to measure beliefs about prevalence. Ten bottle caps represented ten randomly selected members of the community, and the respondent was to remove one bottle cap for each member who was HIV positive.

Columns 4 and 5 of Table 4 show that neither beliefs about the absolute transmission rate, nor beliefs about HIV prevalence were affected by the information campaign. We might expect beliefs about prevalence to be lower in the full intervention arm, as respondents are aware that ART should slow the spread of HIV in the population. This coefficient is small and insignificant, but not negative (Column 5 of Table 4). This may be explained by fact that ART has only been available for the past few years, and adoption has been low, so a reasonable update in beliefs about the spread of HIV might be quite small.

Beliefs about HIV transmission and prevalence are incorrect in all intervention villages. In particular, beliefs about the absolute probability of HIV transmission are much higher than the true value, which, according to the Malawi National AIDS Commission, is approximately $10 \%$ per year. These beliefs are consistent with the overestimates of HIV transmission rates measured by Kerwin (2012) in Malawi, and may be explained by a health education policy which purposely overstates the risk of contracting HIV in an effort to discourage risk taking behavior.

\section{The information intervention reduced discrimination towards ART users}

If we are to believe that a reduction in discrimination between sexual partners has indeed taken place, we ought to see a change in attitudes towards those taking ART drugs. In the model, such discrimination is a result of a rational fear of HIV transmission: some people will reject a potential sexual partner who has been tested for HIV, because such a partner is more likely to be HIV positive and taking ART drugs. The survey measures in Columns 1 and 2 of Table 4 directly address this type of behavior, by asking whether the respondent would reject such a partner, and also whether others would do so. The first measure asks respondents whether they would prefer a sexual partner who is taking ART drugs over one who has never been tested. The second measure asks whether a person taking ART drugs would be able to find a new sexual partner. The fact that the full information intervention caused a significant change in both of these measures suggests that the specific mechanism outlined in our model may apply. 


\subsection{The Effect of the Information Intervention on HIV Testing}

The main outcome of interest, both theoretically and from a policy perspective, is HIV testing. The full information intervention shifted community beliefs about the public benefit of ART drugs (as observed in Section 4.1). As this reduced discrimination towards those who seek ART drugs, we expect to see a higher number of HIV test seekers from full intervention villages. The HIV testing rate is also important for policy, especially in the context of this intervention. The information we provided only addresses the public benefit of ART drugs, so it is likely that any increase in HIV testing will be followed by increased demand for ART. Uptake of ART is important because the medication saves lives and slows the spread of the epidemic.

\section{Administrative data}

We use administrative data from the handwritten ${ }^{28}$ patient registers of 18 health facilities in Zomba District, including all free clinics whose catchment areas include study villages, and all free clinics and hospitals in Zomba Town.

We photographed and digitized HIV testing registers, concealing identifying information due to ethical concerns about privacy. Data on ART use was routinely digitized and provided by Dignitas International, a health care NGO based in Zomba.

We collected HIV testing data for the period covering 2.5 months before the start of the intervention and 4 months after the intervention ended. HIV testing registers at each health facility include fields for the date, gender, age, and address or home village (see Figure 5). This last field allows us to link the HIV test to the patient's village, and in particular, to determine whether that village belongs to one of the study's intervention arms or to the control arm. HIV testing registers also indicate whether the patient was pregnant, whether or not the test was a joint test, the time since the client's last HIV test, and the result of the test. The distance traveled to the facility is calculated from GPS coordinates of the clinics and the study villages, as recorded in census data ${ }^{29}$.

\section{Regression equation}

In the administrative data, we only observe those community members who seek an HIV test, so we are required to use a village-level specification. The primary outcome of interest is the percent of the target population that seeks an HIV test. We construct this outcome measure by first aggregating data on individual HIV tests at the village level, constructing separate variables for tests that took place pre-intervention and post-intervention $^{30}$. The target population for the information campaign consisted of individuals aged 15 to 49 , and we restrict the outcome variable to include only HIV tests sought by members of this age group. Pregnant women are excluded, as they undergo mandatory HIV testing as a part of their antenatal health care. Information from the HIV testing register determines whether a given patient belongs to the target

\footnotetext{
${ }^{28}$ There are two exceptions: Zomba Central Hospital and Matawale Health Facility both have electronic registers. Health facility data was obtained with the permission of the Malawi College of Medicine, as well as the Malawi District Health Office and Zomba Central Hospital

${ }^{29}$ Census data was obtained from the Malawi National Statistics Office

${ }^{30}$ The pre-intervention period covers 2.5 months before the start of the intervention, and the postintervention period covers 4 months after the intervention ended. We ignore all HIV tests that took place during the intervention period, which lasted 22 days.
} 
group, and census data provides the approximate size of the reference population in each village.

In order to identify the causal effect of the information intervention with high statistical power, we perform an ANCOVA regression at the village level, controlling for pre-intervention levels of the outcome variable. This estimator is unbiased in a randomized study, and has lower variance than either a simple regression of post-intervention testing levels on treatment, or a difference-in-difference estimator (McKenzie, 2012).

As stated above, the dependent variable is the percent of the target population who sought an HIV test post-intervention. Covariates include the pre-intervention testing rate, and other village-level covariates determined from the Malawi National Census, GPS coordinates, and the survey. The full set of village-level and pre-intervention covariates is listed in Table 2. We also control for the stratification variables: village population and distance to each health facility.

This intervention may be subject to spillovers. Indeed, $60 \%$ of the villages in the study are within 1 kilometer of another study village. We therefore include a specification which controls for nearby study villages, nearby intervention villages, and nearby full intervention villages, considering villages within 1 kilometer (based on GPS coordinates). Because the intervention was randomly assigned, including these regressors together should not introduce endogeneity, and will remove the downward bias caused by spillovers from full intervention villages to other villages.

Our regression specification is as follows:

Percent HIV tested $_{j}=\alpha+\beta_{1}\left(P_{j}+F_{j}\right)+\beta_{2} F_{j}+\gamma_{0} N_{j}+\gamma_{1}\left(N_{P j}+N_{F j}\right)+\gamma_{2}\left(N_{F j}\right)+\delta^{\prime} \chi_{j}+\epsilon_{j}$.

$P_{j}$ and $F_{j}$ are indicators for the partial and full intervention arms respectively, $\chi_{p}$ is a vector of village-level covariates including the pre-intervention testing level (see Table 2 for a complete list of village-level covariates). $N_{j}$ is the total number of study villages within one kilometer, $N_{P j}$ is the number of partial intervention villages within one kilometer, and $N_{F j}$ is the number of full intervention villages within one kilometer. Standard errors are robust.

Because $P_{j}$ and $F_{j}$ are randomly assigned, $\mathbb{E}\left(\epsilon_{j} \mid P_{j}, F_{j}\right)=0$, and the OLS estimates $\hat{\beta}_{1}$ and $\hat{\beta}_{2}$ are unbiased.

\section{The information intervention increased HIV testing}

Providing information on the private benefit of ART drugs does not appear to significantly increase HIV testing. The effect of the partial intervention appears to be positive but insignificant, as shown in Table 6. A show of hands during the information intervention suggested that the private benefits of ART were relatively well known, which may explain a modest or null effect on HIV testing. Even if some were surprised to learn that ART is available and has large private benefits, this may not be a large enough incentive to test for a person who does not know his status. Such a person will only benefit from ART if he tests positive for HIV, and the costs of testing, including discrimination, may be high.

Providing information on the public benefit of ART drugs has a significant effect on HIV testing. The full information intervention caused a significant increase in the total number of HIV tests relative to the partial intervention, as shown in Table 6. 
Controlling for spillovers, the estimated effect size is $27 \%$, measured over a four-month period. Spillovers from the full intervention arm are positive and significant. Such spillovers may be due to the spread of information, or a perceived change in attitudes towards those who seek an HIV test.

Let us turn to the epidemiological literature to put this magnitude of HIV testing into some context. Granich et al. (2009) provide a mathematical model for the relationship between HIV testing and the spread of HIV, taking into account the fact that ART drugs greatly reduce transmission. The intervention increased annual testing rates among sexually active individuals from approximately $9 \%$ to $12 \%$, which according their model should avert at least two new HIV infections per primary infection. While this is based on a model which abstracts from many behavioral considerations, it is worth noting that at low levels of HIV testing, a small increase has the potential to have large epidemiological implications.

\section{The choice of testing location is linked to beliefs about the public benefit of ART}

Before the intervention, in the average village, $49 \%$ of those seeking an HIV test traveled at least two kilometers further than necessary from their home villages. This may be indicative of stigma or discrimination, because traveling far from home is costly, but reduces the likelihood of being seen by friends, neighbors, and potential sexual partners.

In general, individuals seeking an HIV test may travel to far away clinics for reasons other than to avoid being seen, such as differences in clinic quality or wait time. However, HIV testing is free, and the procedures are identical at all clinics. Wait times do not vary much, especially compared with the time spent traveling to the clinic; those who test far away travel, on average, an extra nine kilometers. Finally, if far away testing is fully explained by reasons other than discrimination, we should not observe any link between beliefs about the public benefits of ART and the choice of testing location.

We say that an HIV test takes place far away if it takes place at least two kilometers further than the client's nearest health facility ${ }^{31}$, and investigate the effect of a shift in beliefs about the public benefit of ART on testing location. We again run an OLS regression with specification (16), restricting the sample to intervention villages and comparing the partial intervention to the full intervention. The dependent variable is the number of nearby or far away HIV tests; Columns 1 and 2 of Table 6 show the results of these three regressions. A shift in beliefs may cause an increase in both nearby and far away testing: the coefficients are positive but imprecisely estimated.

In order to understand the full intervention's ambiguous effect on testing location, we turn to the predictions of the model. The model predicts a specific relationship between the choice of HIV testing location and the distribution of beliefs about ART in the community, based on the link between those beliefs and stigma. In particular, a moderate shift in beliefs about the ART prevention parameter $\rho$, which corresponds to a moderate reduction in stigma, could increase both the number of people who test near their home village and the number who test far away. Meanwhile, a large shift in beliefs, which corresponds to a large reduction in stigma, should only increase the number of nearby tests, as people become less afraid of being seen to seek an HIV test.

\footnotetext{
${ }^{31}$ See the Appendix for results using other definitions.
} 
We define high beliefs as beliefs ${ }^{32} \hat{\rho} \geq 0.95$, for the simple reason that this threshold approximately matches the true value of $\rho$. From a policy perspective, it is useful to know the effects of shifting beliefs to the true value of $\rho$, and whether this is sufficient to cause a reduction in stigma. As the majority of individuals in partial intervention villages hold beliefs $\hat{\rho}=0$, we define moderate beliefs to be $0<\hat{\rho}<0.95$.

On average, the full information intervention shifted beliefs from low beliefs $\hat{\rho}=0$ to either moderate or high beliefs, as shown in Figure 7. The distribution of beliefs in a given village can be described by three numbers: the proportion of the population who hold low beliefs, moderate beliefs, and high beliefs.

We analyze the following OLS specification.

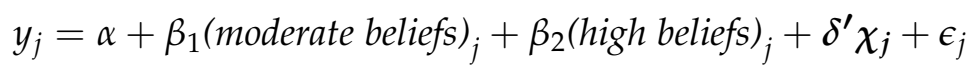

$y_{j}$ is set equal to either the percent of the target population in village $j$ that seeks an HIV test nearby, or the percent of the target population in village $j$ that seeks an HIV test far away from their home village. The regressors (moderate beliefs) ${ }_{j}$ and (high beliefs) represent the proportion of the village that has moderate or high beliefs, as estimated from survey responses. These regressors are not exogenous, and we must interpret the estimated coefficients $\hat{\beta}_{1}$ and $\hat{\beta}_{2}$ as correlations between the level of community beliefs and the number of HIV tests either nearby or far away. $\chi_{p}$ is a vector of village-level covariates $^{33}$. Standard errors are robust.

Consistent with the predictions of the model, high beliefs about the public benefit of ART are associated with a significant increase in the number of nearby tests, and an (insignificant) decrease in the number of far away tests, as seen in Columns 3 and 4 of Table 6.

\section{HIV testing increases if the public benefit of ART is common knowledge}

The theory predicts that HIV testing decisions should depend on the level of discrimination in the community, which in turn depends on community-level beliefs about the public benefit of ART. This differs sharply from predictions based on a model of social preferences, in which one's own beliefs matter most, or a model of household bargaining, in which one's spouse's beliefs are important. In our model, in order for beliefs to affect the testing decision, the beliefs of the community must be common knowledge.

In order to test this prediction, we measured perceived beliefs of the community. That is, we asked respondents what they thought most people in their respective communities believed about the public benefit of ART. Once a respondent had fully understood the infographic in Figure 6, she was asked which option that her spouse would choose, and which option most members of the community would choose.

We regress self-reported HIV testing on this measure of perceived beliefs. We use

\footnotetext{
${ }^{32}$ These beliefs are elicited in the survey using the infographic in Figure 6. Note that this beliefs measure is discrete, and the selection $\hat{\rho}=0.95$ is closest to the true value of $\rho=0.96$.

${ }^{33}$ See Table 2 for a complete list of village-level covariates.
} 
the specification

$$
\begin{array}{r}
\text { HIV test } t_{i j}=\alpha+\beta_{1}{\text { (respondent has high beliefs })_{i}+\beta_{2}(\text { spouse has high beliefs })_{i}+} \\
\beta_{3}(\text { community has high beliefs })_{i}+\delta^{\prime} \chi_{i j}+\epsilon_{i j}
\end{array}
$$

where the dependent variable is an indicator for respondent $i$ in village $j$ reporting an HIV test post-intervention. The three regressors of interest are the respondent's own beliefs, her perception of her spouse's beliefs, and her perception of the community's beliefs. These three variables are indicators for high beliefs about the public benefit of ART, $\hat{\rho}>0.95$. The set of covariates $\chi_{i j}$ includes individual- and village-level controls ${ }^{34}$. Standard errors are clustered at the village level.

As predicted, Table 7 shows that survey respondents are much more likely to report an HIV test post-intervention if they perceive the community to have high beliefs about the public benefit of ART drugs. The respondent's own beliefs and perceived spouse's beliefs do not play a significant role.

\subsection{The Effect of the Intervention on Initiation of ART Drugs}

In this section, we investigate the effect of the information intervention on ART initiation within four months. Within this time frame, it would be unlikely for a person to both seek an HIV test and begin treatment. Most ART initiators will be individuals who tested positive before the intervention but did not seek ART. These individuals are likely to have underestimated the private benefits or availability of ART, as they did not begin treatment. They are also likely to have faced relatively low costs of testing, including low levels of discrimination, since they were willing to seek an HIV test without being fully aware of the availability ${ }^{35}$ of high quality treatment ${ }^{36}$.

Access to ART drugs is an enormous benefit of HIV testing for those who are infected. In the model, ART is assumed to be the only motivation for testing, and we assume that every patient who is tests positive for HIV seeks ART ${ }^{37}$. This is justified when we consider only the partial and full intervention arms, as we provide information on the high private benefits and availability of ART in both interventions. However, preintervention, and in the control arm, some individuals may underestimate the private benefits or availability of ART drugs.

There are reasons to believe that both the partial and full intervention may increase ART initiation among this type of HIV positive individual. First, those who have tested positive in the past but not initiated ART are likely to have underestimated the private benefits or availability of the drugs, and will be particularly susceptible to the messages conveyed in the partial intervention. Second, if discrimination from sexual partners is a barrier to ART drugs over and above the barrier they pose to HIV testing, the full

\footnotetext{
${ }^{34}$ Individual-level controls are age, gender, whether the person is pregnant, married, has regular partner, employed, primary school educated, secondary school educated, has livestock, and has a brick house.. Village-level controls are covariates listed in Table 2 and community educator fixed effects.

${ }^{35}$ In the past, access to ART drugs was restricted to individuals whose symptoms had progressed beyond a certain point, or who had a low CD4 count (a measure related to HIV viral load). It is possible that patients who tested positive but did not initiate treatment did not qualify for ART at the time of testing.

${ }^{36}$ In the language of the model, such patients would have had a low $y_{a}$ parameter in order to seek a test without understanding the large benefits of treatment $v$.

${ }^{37}$ In our data, the rate of ART initiation is approximately $80 \%$ of the rate of testing positive for HIV.
} 
intervention may further increase ART uptake among those who have already tested positive.

However, as previously mentioned, the type of person who has already sought an HIV test, even without the intention to initiate treatment, must not have perceived discrimination as a significant barrier. It could be that such patients were not concerned about discrimination from potential partners, or were already visibly infected, and so did not provide any additional signal to the community by seeking an HIV test. These factors imply that the full intervention, in which discrimination is reduced, may not have a large effect on ART initiation over and above the partial intervention.

We again use specification (16), with the percent of the target population initiating ART drugs as the dependent variable. As shown in Table 8, both the partial and full intervention significantly increase ART initiation in the four months following the intervention. The full intervention does not appear to increase ART initiation over and above the partial intervention, suggesting that for this group of patients, who had likely already sought an HIV test, a lack of understanding of the private benefits or availability of ART drugs was a much larger barrier than discrimination from sexual partners.

\subsection{Sexual Behavior}

Providing information on the public benefit of ART, and the subsequent reduction in discrimination from potential sexual partners may lead to risk compensation in the context of sexual behavior. However, as shown in Section 3, this is not necessarily the case. A reduction in discrimination increases HIV testing, and the net effect on consent to risky sex is ambiguous.

At the onset of the HIV epidemic, the behavioral response in southern Africa was limited $^{38}$ (Oster, 2012), so even a return to pre-epidemic behavior would imply a fairly small increase in risky sex. However, Friedman (2012) finds that the arrival of ART lowered the perceived cost of contracting HIV, which in turn increased pregnancy rates among young women. This suggests that beliefs about the private benefits of ART affect risk taking; in this section we investigate the effect of beliefs about the public benefit of ART.

We regress self-reported measures of sexual behavior on intervention arm, following specification (15). The dependent variables are the number of sex acts and number of condoms used, both recalled by respondents over the past seven days.

The number of sex acts is insignificantly higher, and the number of condoms used is insignificantly lower in the full intervention group (Table 10).

While the increase in risk taking is statistically insignificant, the magnitude of the coefficient is not negligible. Before concluding that this is a harmful effect of the information campaign, it is worth noting that rational agents may be expected to increase their sexual interactions in response to the information. Risk compensation may come with large benefits including, for example, being able to start a family.

Self-reported measures are often subject to desirability bias, and if this bias is equal in both partial and full intervention groups, it should not introduce bias into our OLS estimates. However, there is reason to believe that the full intervention may have affected desirability bias by making it seem less unreasonable to report risky sex with

\footnotetext{
${ }^{38}$ Low non-HIV life expectancy (Oster, 2012) and a high perceived cost of protective behavior (for example, in terms of fertility) led to high levels of risky sex, even in the midst of an HIV epidemic.
} 
a person who has been tested and/or treated for HIV. If this is the case, our estimates will be biased, and will overestimate the extent to which the full intervention increased risk-taking.

Finally, if we assume that the increase in risk taking was among those who either tested negative, or are taking ART, the level of risk compensation we observe is inconsequential in terms of HIV transmission ${ }^{39}$ compared with the preventative strength of ART drugs. There would need to be a minimum 25-fold increase in unprotected sexual activity to offset the $96 \%$ reduction in HIV transmission associated with $\mathrm{ART}^{40}$.

\subsection{Other Potential Mechanisms}

We claim that the effects of the information experiment on HIV testing are due to a reduction in discrimination towards those who seek an HIV test. However, other models may generate similar predictions. For example, both social preferences and household bargaining would predict an increase in HIV testing in response to new information about the public benefits of ART drugs. We will attempt to rule these out as first order explanations for our results.

Social costs are not the only potential barrier to HIV testing and treatment. For example, the psychic costs of testing may be quite large. Oster et al. (2013) propose an optimal expectations model to explain low uptake of a medical test for Huntington's Disease. This type of model, in which individuals prefer to choose optimistic beliefs about the future rather than learn the truth, may also partly explain low HIV testing rates. However, such behavior is unlikely to be affected by information on the public benefit of ART, conditional on the private benefits being known. For this reason, we restrict attention to mechanisms which are plausibly linked to our intervention through a social component of behavior change.

\section{Social preferences}

Altruism is an obvious alternative mechanism by which information on the public benefit of ART might increase HIV testing. People may derive utility from protecting their sexual partners from HIV. If this were the primary mechanism, we would expect to see self-reported testing increase in one's own beliefs about the public benefits of ART, as opposed to the beliefs of others. As shown in Table 7, this does not seem to be the case. Additionally, while altruism may cause either nearby or far away testing to increase, it does not explain the pattern observed in Table 6; high beliefs about the public benefit of ART are linked specifically to nearby testing.

This is not to suggest that individuals are not altruistic, or that altruism does not play a role in HIV testing. It is plausible that a person who is altruistic towards his partner is also less likely to be HIV positive, as he might use condoms and have fewer concurrent partners. In addition, an altruistic person may choose to test even without

\footnotetext{
${ }^{39}$ Risk compensation may be of concern for other reasons, for example, the spread of other sexuallytransmitted infections which ART does not prevent. Gong (2014) and Baird et al. (2014) find an increased risk of sexually-transmitted infection after a person tests positive for HIV.

${ }^{40}$ Let $\tau$ be the HIV-transmission rate. The probability of avoiding infection in an interaction with an HIV-positive person is $1-\tau$, or, if the person is taking ART, $1-0.04 \tau$. We are interested in solving for the proportional increase in sexual interactions $x$ which equalizes risk: $(1-\tau)=(1-0.04 \tau)^{x}$. For any $\tau \in(0,1)$, this is solved by $x>25$.
} 
knowledge of the public benefit of ART, in order to make informed choices about the risk of transmitting the virus.

\section{Household bargaining}

Household bargaining has been shown to play a role in family planning decisions (Ashraf et al., 2013). There are many reasons to believe that HIV testing decisions are also subject to household bargaining, and this suggests an alternative explanation for the increase in HIV testing we observed. In particular, a person who is aware of the fact that ART reduces HIV transmission may put greater pressure on her spouse to get tested and treated for HIV. In this case, we would expect the testing decision to be strongly linked to one's spouse's beliefs about the benefits of ART. Table 7 shows that this is not the case.

A model of household bargaining would also predict an increase in joint testing. A person has little to gain by putting pressure on her partner to seek an HIV test if he is not intrinsically motivated to get treated. The results of the test are private, and he can simply report a negative test result. However, if a couple is tested jointly, the results are seen by both, which means that each member of the couple can exert additional pressure on the other to seek ART if necessary. Column 1 of Table 10 shows that the effect of the full information campaign on joint testing is insignificant, and does not explain the overall increase in HIV testing. Survey results indicate thatpartial and full intervention villages are similar in terms of HIV tests reported due to pressure, preference for joint testing over private testing, and relative willingness to pay for joint and private testing (see Columns 2, 3, and 4 of Table 10).

Household bargaining does not seem to explain the increase in HIV testing we have seen as a result of the information campaign. It may nevertheless play some role in HIV testing decisions. Most respondents report a strong preference for joint testing, and approximately $20 \%$ of HIV tests in the clinic data are joint tests. Those with enough bargaining power to pressure their partners to test jointly may have already been motivated to do so before learning that ART would protect them from transmission. For example, joint testing allows couples to make informed decisions about condom use and family planning, and form accurate expectations about the future.

\section{Conclusion}

This study identifies a particular type of social barrier to HIV testing: statistical discrimination between potential sexual partners. HIV testing disproportionately benefits those infected with the virus, and is therefore a signal of underlying risk. An individual's testing decision may be observed by members of the community, and some community members reject potential sexual partners who are known to have been tested.

This example of statistical discrimination is based on misinformation. Most are unaware of the fact that an HIV-positive person who is tested and treated for HIV is much less contagious, due to the public benefit of ART drugs. Providing information about the public benefit of ART reduces stigma, and increases HIV testing by $27 \%$. Villages with high beliefs about the public benefit of ART drugs after the intervention see a large increase in the number of HIV tests sought at nearby clinics, but no increase in HIV testing at far away clinics. In addition, the testing decision appears to be based 
on common knowledge of the community's beliefs about ART, and not one's own beliefs. These results suggest that it is possible to diminish this form of discrimination, by shifting the incorrect beliefs upon which it is based.

If the increase in HIV testing we observed was indeed due to a reduction in communitylevel discrimination, the effect should persist. Uptake of ART drugs should also increase. In addition, the theory suggests that the intervention targeted those who care most about the number of sexual prospects. Individuals who prefer to have many potential sexual partners are also most at risk of contracting and spreading HIV. Longrun data on HIV incidence will allow us to investigate the effect of the intervention on the spread of the virus.

This work may be relevant to other contexts in which a technology is under-adopted due to stigma. In particular, if a technology disproportionately benefits a stigmatized group, it may be under-adopted, as individuals know it will signal their underlying type. For example, a person who suffers from mental illness may be reluctant to seek psychiatric care, for fear his friends or employer might find out. Stigma, in many contexts, may be explained by statistical discrimination; employers might believe that the mentally ill are less productive workers. This situation leads to a bad equilibrium: the employee's mental illness goes untreated, and he is less productive than he would be if he were receiving treatment. Of course, one solution is to help the employee obscure the signal, by ensuring privacy of psychiatric care. However, if statistical discrimination is based on incorrect beliefs, providing correct information to the public, or in this example, to employers, should eliminate the stigma equilibrium.

\section{Information as policy}

A community-level information campaign on the public benefit of ART drugs is an inexpensive way to reduce discrimination towards people who are HIV positive, and increase HIV testing. A policy which reduces discrimination directly is more attractive than one which helps HIV test-seekers conceal their decision to test or their motivation for testing. For example, while monetary incentives increase HIV testing, in order to be effective in the face of stigma, they must be paid repeatedly to the entire community. This might make monetary incentives for ART use infeasible. A reduction in the level of discrimination should affect all health seeking behavior, and have permanent effects.

Many countries, including Malawi, are moving towards policies of universal testing and treatment. We showed that providing information on the public benefit of ART increases HIV testing, and that even providing basic information on the private benefits and availability of ART drugs greatly increases uptake for those who have already tested HIV positive. Our results suggest that community outreach, and the provision of accurate, actionable information should be a part of any policy to increase health seeking behavior among HIV positive individuals.

While our experiment took place in Malawi, there is reason to believe its policy lessons apply elsewhere. Southern Malawi is representative of sub-Saharan Africa in many ways. HIV prevalence is high (above 10\%), ART drugs are widely available at rural clinics, and the population is concentrated in rural villages, in which health seeking behavior is often observable. Our model of discrimination between sexual partners is based on rational behavior and does not depend on cultural norms, therefore it might well apply in communities outside of our study area. 
A scaled policy intervention should have two important features: information on the public benefit of ART should be provided at the community-level, to ensure common knowledge, and the information should be provided in a credible way, for example, by trusted health authorities. These aims may be achieved at very low cost, for example by employing existing community health workers, or even using billboards and radio messages; this intervention is a highly cost effective strategy to increase HIV testing and treatment.

It is not sufficient to rely on learning and information sharing to shift beliefs over time, because in the presence of stigma or discrimination, both of these activities will be hampered. Under-adoption of ART drugs means that most people do not learn of their effects first hand, and stigma makes people reluctant to discuss these effects with social contacts. Additionally, those who are infected cannot transmit the information credibly, as they stand to benefit from a shift in community beliefs about ART drugs. In the absence of an information campaign, discrimination and social stigma allow incorrect beliefs to persist.

Our results suggest that providing precise information on the effect of ART drugs on HIV transmission is an effective way to reduce discrimination towards people living with HIV. Some public health campaigns aim to inflate perceptions of HIV risk by distorting or hiding facts about transmission from the public, in an effort to reduce risk taking. This study suggests that such policies may have unintended consequences; they exacerbate discriminatory behavior and inhibit health seeking behavior. Informed communities have higher rates of HIV testing and treatment, which improves the lives of those who are infected, and serves the community as a whole by reducing the spread of the virus.

\section{References}

Altonji, J. G. and Pierret, C. R. (2001). Employer learning and statistical discrimination. The Quarterly Journal of Economics, 116(1):313-350.

Angotti, N., Kaler, A., and Ramaiya, A. (2016). 'you cannot believe your eyes': Gendered dimensions of art anxiety in rural malawi. Presented at Population Association of America Conference, Washington, DC.

Arrow, K. (1973). The theory of discrimination. Discrimination in labor markets, 3(10):333.

Ashraf, N., Field, E., and Leight, J. (2013). Contraceptive access and fertility: The impact of supply-side interventions. Working Paper.

Baird, S., Chirwa, E., McIntosh, C., and Özler, B. (2010). The short-term impacts of a schooling conditional cash transfer program on the sexual behavior of young women. Health economics, 19(S1):55-68.

Baird, S., Gong, E., McIntosh, C., and Ozler, B. (2014). The heterogeneous effects of hiv testing. Journal of Health Economics, 37:98-112. 
Baird, S. J., Garfein, R. S., McIntosh, C. T., and Özler, B. (2012). Effect of a cash transfer programme for schooling on prevalence of hiv and herpes simplex type 2 in malawi: a cluster randomised trial. The Lancet, 379(9823):1320-1329.

Bandiera, O., Buehren, N., Burgess, R., Goldstein, M., Gulesci, S., Rasul, I., and Sulaiman, M. (2012). Empowering adolescent girls: Evidence from a randomized control trial in Uganda. Working Paper.

Benabou, R. and Tirole, J. (2011). Laws and norms. Working Paper.

Besley, T. and Coate, S. (1992). Understanding welfare stigma: taxpayer resentment and statistical discrimination. Journal of Public Economics, 48(2):165-183.

Chinkhumba, J., Godlonton, S., and Thornton, R. (2012). The demand for medical male circumcision. American Economic Journal: Applied Economics, 6(2):152-177.

Coate, S. and Loury, G. C. (1993). Will affirmative-action policies eliminate negative stereotypes? The American Economic Review, pages 1220-1240.

Cohen, M. S., Chen, Y. Q., McCauley, M., Gamble, T., Hosseinipour, M. C., Kumarasamy, N., ..., and Fleming, T. R. (2011). Prevention of HIV-1 infection with early antiretroviral therapy. New England Journal of Medicine, 365:493-505.

Delevande, A. and Kohler, H.-P. (2009). Subjective expectations in the context of HIV / AIDS in Malawi. Demographic Research, 20(31):817-875.

DHS (2010). Malawi DHS, 2010 - Final report.

Duflo, E., Dupas, P., and Kremer, M. (2012). Education, HIV, and early fertility: Experimental evidence from Kenya. Working Paper.

Dupas, P. (2011). Do teenagers respond to HIV risk information? Evidence from a field experiment in Kenya. American Economic Journal: Applied Economics, pages 1-34.

Friedman, W. (2012). Antiretroviral drug access and behavior change. Working Paper.

Godlonton, S., Munthali, A., and Thornton, R. (2011). Behavioral response to information? Circumcision, information, and HIV prevention. Bureau for Research and Economic Analysis of Development Working Paper.

Goffman, E. (1963). Stigma: Notes on the management of spoiled identity. Prentice-Hall.

Gong, E. (2014). HIV testing and risky sexual behaviour. Working Paper.

Granich, R. M., Gilks, C. F., Dye, C., Cock, K. M. D., and Williams, B. G. (2009). Universal voluntary HIV testing with immediate antiretroviral therapy as a strategy for elimination of HIV transmission: a mathematical model. The Lancet, 373(9657):4857.

Helleringer, S., Kohler, H.-P., and Kalilani-Phiri, L. (2009). The association of hiv serodiscordance and partnership concurrency in likoma island (malawi). AIDS (London, England), 23(10):1285. 
Hoffmann, V., Fooks, J. R., and D. Messer, K. (2014). Measuring and mitigating hiv stigma: A framed field experiment. Economic Development and Cultural Change, 62(4):701-726.

Jalan, J. and Somanathan, E. (2008). The importance of being informed: Experimental evidence on demand for environmental quality. Journal of Development Economics, 87(1):14-28.

Kerwin, J. (2012). Rational fatalism: non-monotonic choices in response to risks. Working Paper.

Kerwin, J. T. (2014). The effect of HIV infection risk beliefs on risky sexual behavior: Scared straight or scared to death? Working Paper.

Madajewicz, M., Pfaff, A., Van Geen, A., Graziano, J., Hussein, I., Momotaj, H., Sylvi, R., and Ahsan, H. (2007). Can information alone change behavior? Response to arsenic contamination of groundwater in bangladesh. Journal of Development Economics, 84(2):731-754.

Mahajan, A. P., Sayles, J. N., Patel, V. A., Remien, R. H., Ortiz, D., Szekeres, G., and Coates, T. J. (2008). Stigma in the HIV/AIDS epidemic: A review of the literature and recommendations for the way forward. AIDS, 22(2):S67.

McKenzie, D. (2012). Beyond baseline and follow-up: The case for more $\mathrm{T}$ in experiments. Journal of Development Economics, 99(2):210-221.

Moffitt, R. (1983). An economic model of welfare stigma. The American Economic Review, pages $1023-1035$.

Ngatia, M. (2011). Social interactions and individual reproductive decisions. Technical report.

Oster, E. (2012). Hiv and sexual behavior change: Why not africa? Journal of Health Economics, 31(1):35-49.

Oster, E., Shoulson, I., and Dorsey, E. R. (2013). Optimal expectations and limited medical testing: Evidence from Huntington disease. American Economic Review, 103(2):804-30.

Peski, M. and Szentes, B. (2013). Spontaneous discrimination. The American Economic Review, 103(6):2412-2436.

Thornton, R. L. (2008). The demand for, and impact of, learning HIV status. The American Economic Review, 98(5):1829.

WHO (2013). Report on the global AIDS epidemic. 
Figure 1: Study Area
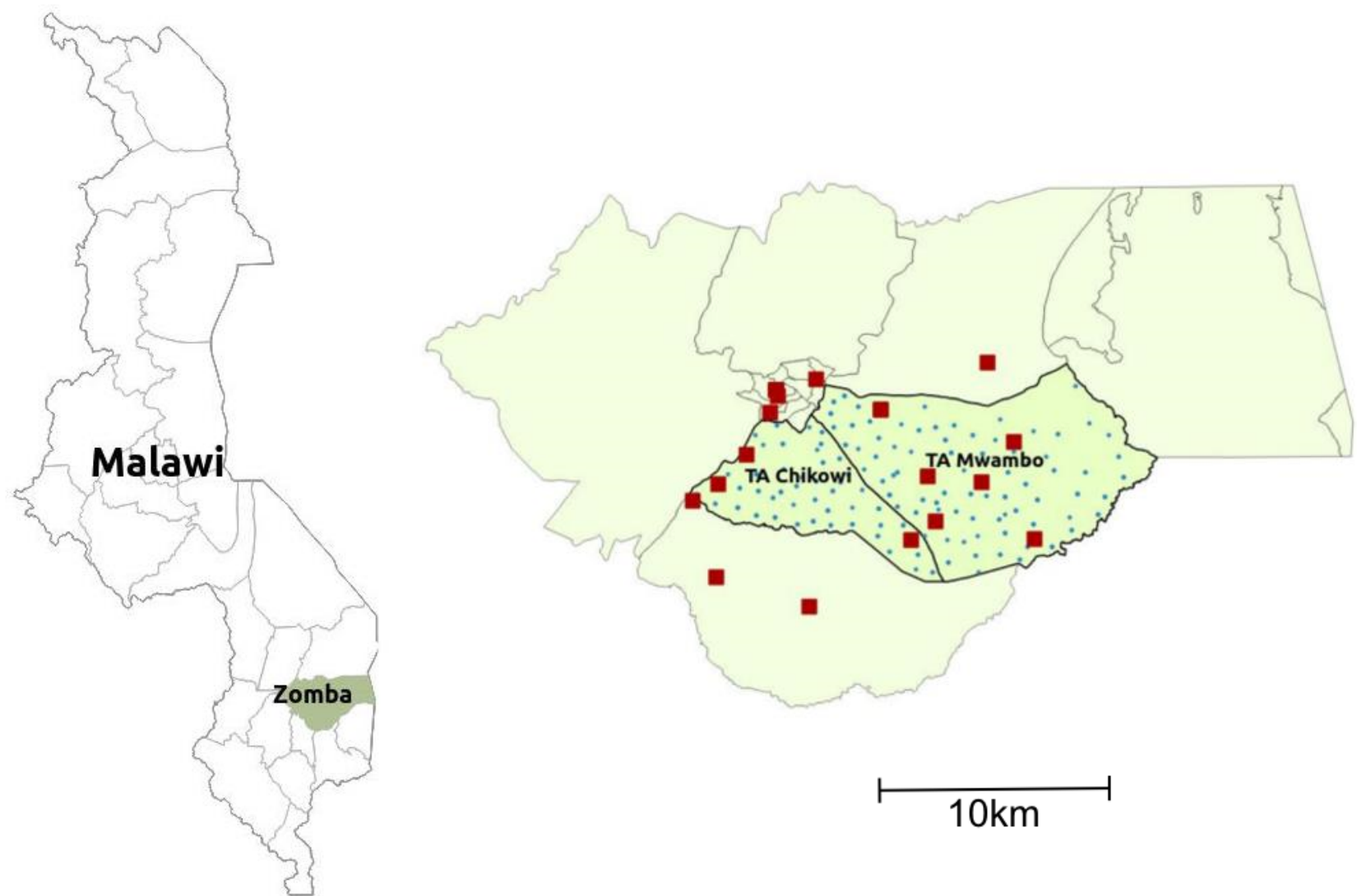

Notes: The study included 244 villages in Zomba District, Malawi, represented by blue dots. Administrative data was obtained from 18 health facilities, represented by red squares. 
Figure 2: Discrimination and HIV Testing Location

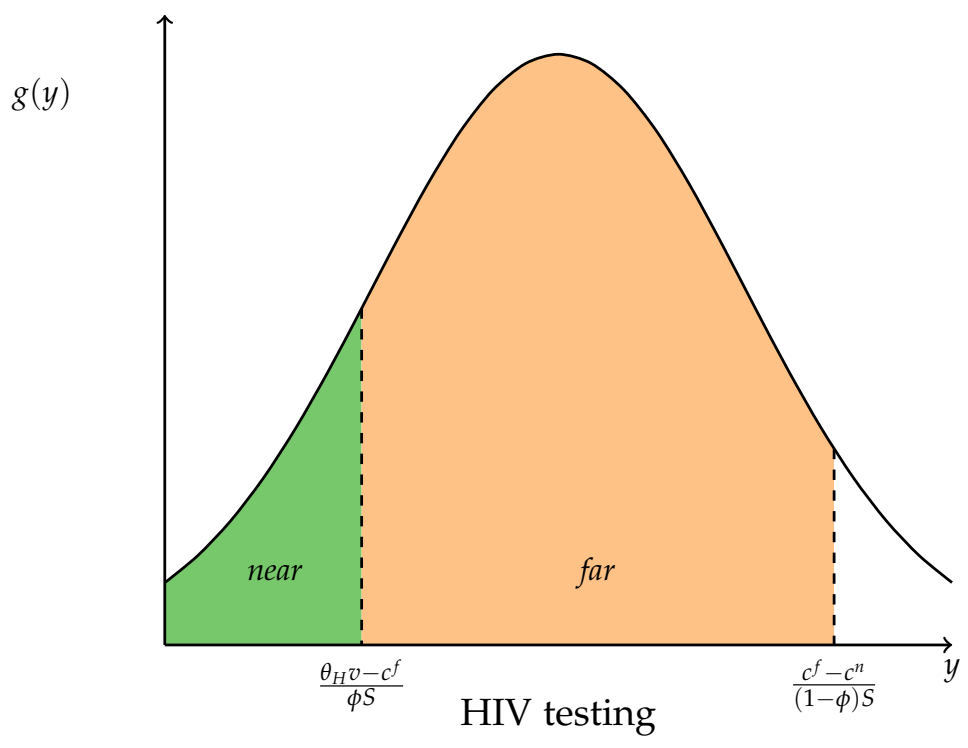

(a) High discrimination

(b) Moderate discrimination

(c) Low discrimination
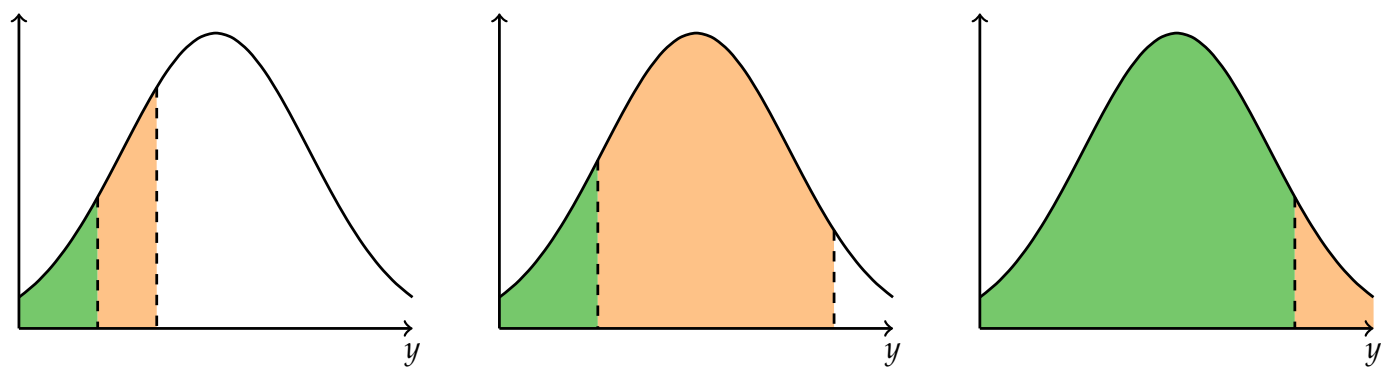

Notes: This figure illustrates the proportion of agents $a \in \mathcal{A}$ who seek nearby and far away testing, respectively. The curve $g(y)$ is the probability density function for the benefit of a relationship, $y_{a}$. To the left of the first threshold, agents seek a nearby HIV test. Between the first and second thresholds, agents seek a far away test. To the right of the second threshold no HIV test is sought. These thresholds shift up as discrimination $S$ decreases; a lower level of discrimination implies a higher total number of tests and a higher number of nearby tests. The effect of a shift in the level of discrimination on the number of far away tests is in general ambiguous; in this example far away testing first increases and then decreases. As $S \rightarrow 0$, the number of far away tests decreases to 0 . 
Figure 3: Infographic for Partial Intervention
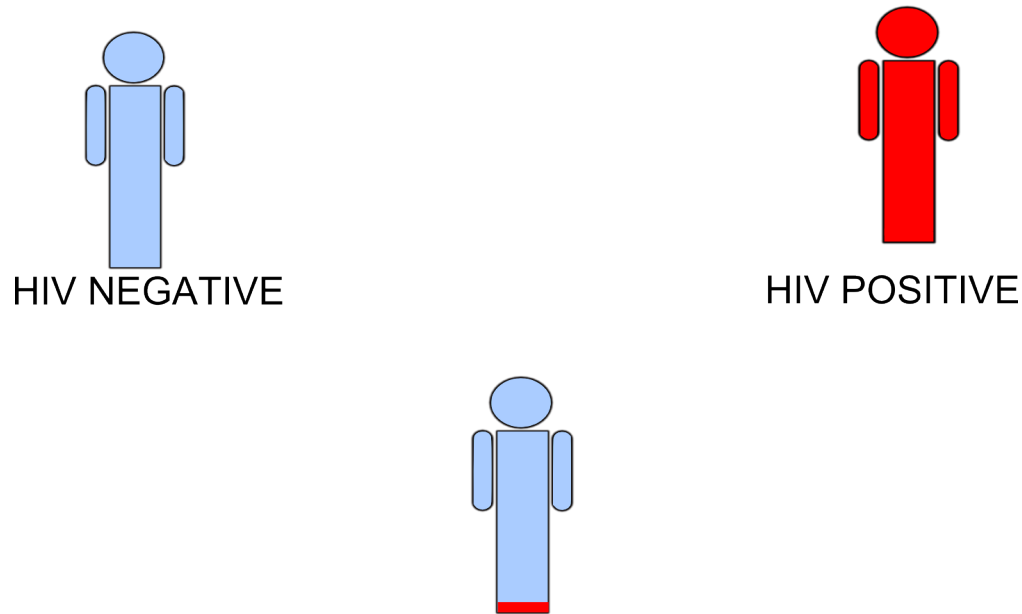

HIV POSITIVE + ART

Notes: The amount of red represents the viral load. Equivalent infographics with an HIV-positive woman were also shown at each meeting.

Figure 4: Infographic for Full Intervention
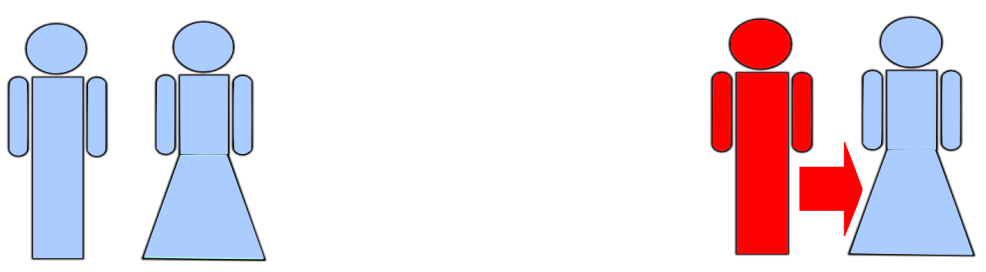

HIV NEGATIVE

HIV POSITIVE

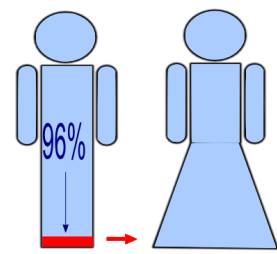

HIV POSITIVE + ART

Notes:The amount of red represents the viral load. The size of the arrow represents HIV transmisison risk. Equivalent infographics with an HIV-positive woman were also shown at each meeting. 
Figure 5: HIV Testing Register

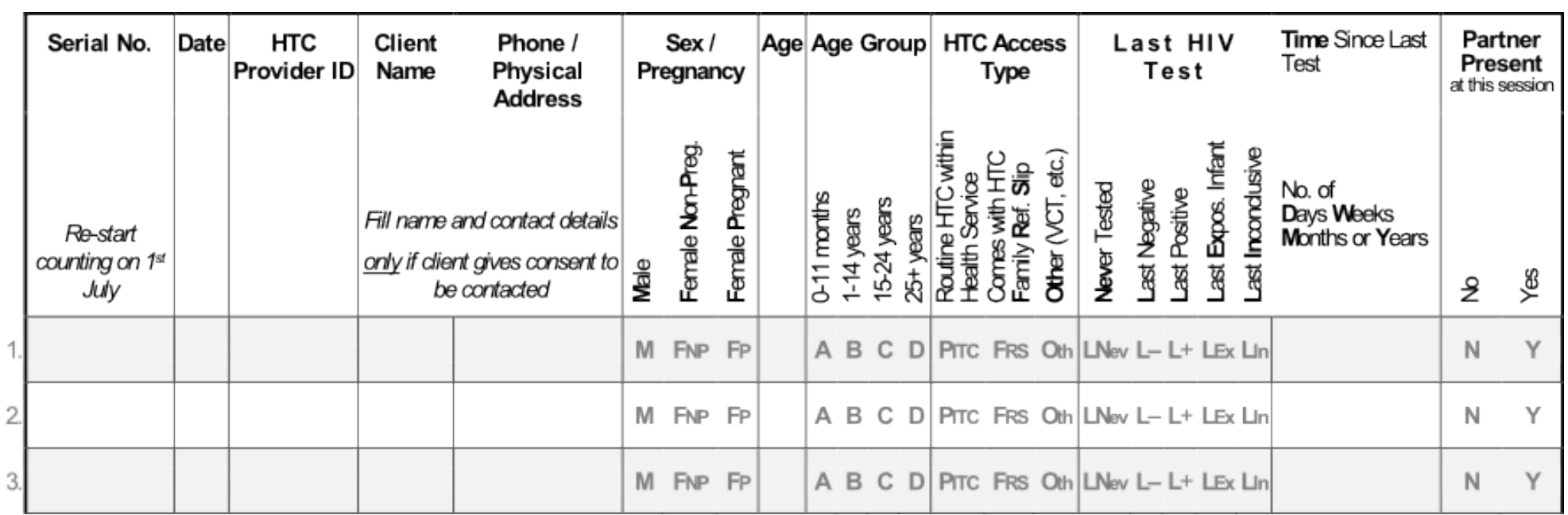


Figure 6: Infographic to Elicit Beliefs

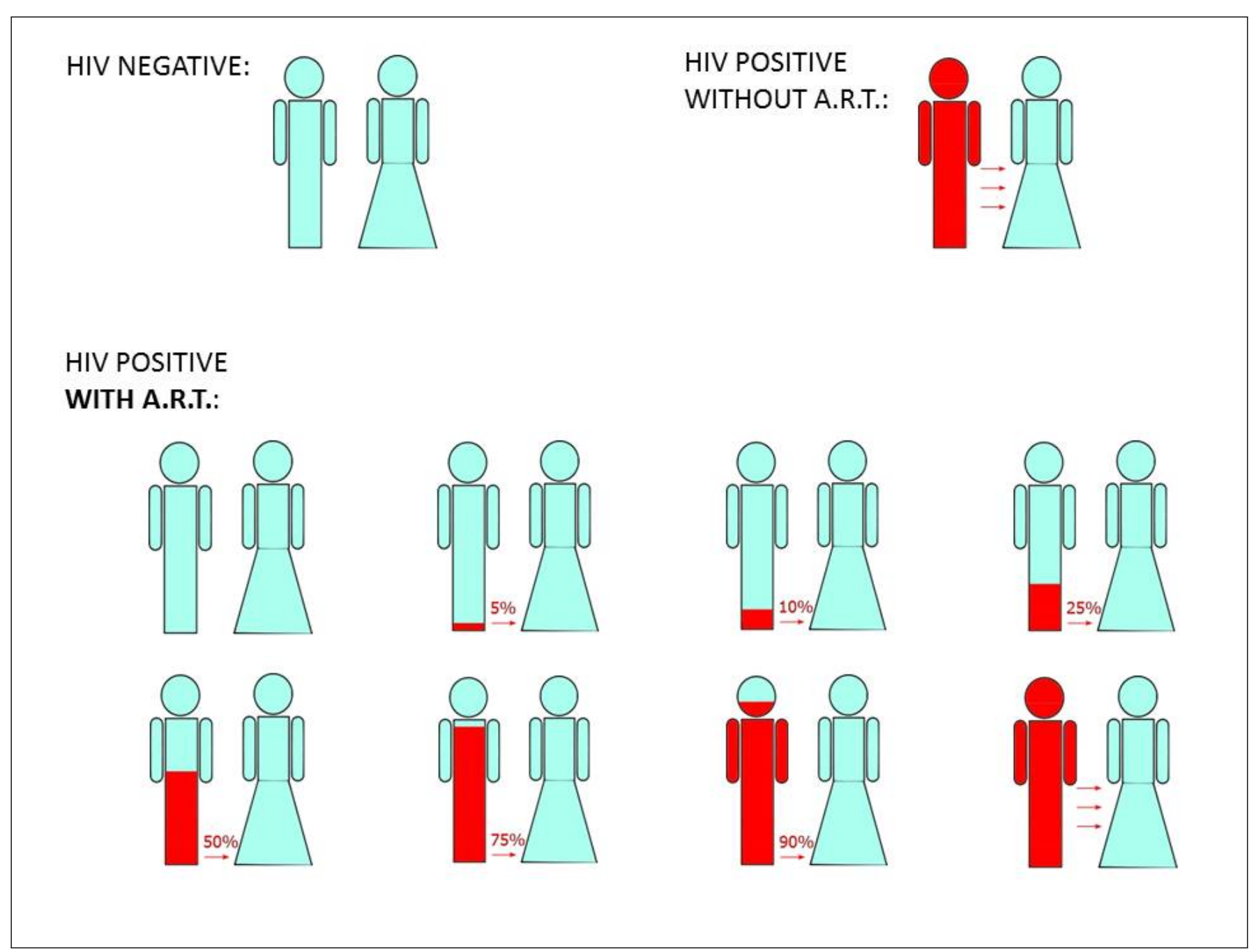

Notes: Respondents were asked to state their beliefs about the relative rate of HIV transmission for a person taking ART drugs by selecting one of eight options. The top left corresponds to the belief that an infected person taking ART drugs is not at all contagious. The bottom right corresponds to the belief that an infected person taking ART drugs and an infected person not taking ART drugs are equally contagious. 
Figure 7: Beliefs $\hat{\rho}$ (ART Prevention Parameter)

(a) Partial intervention

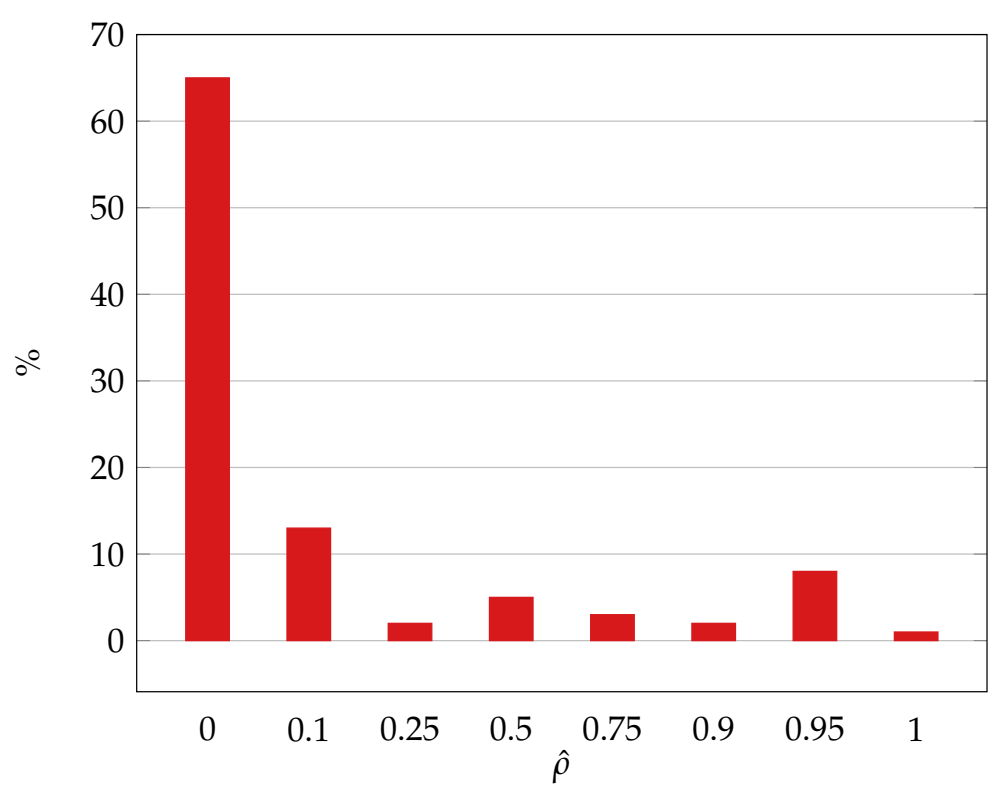

(b) Full intervention

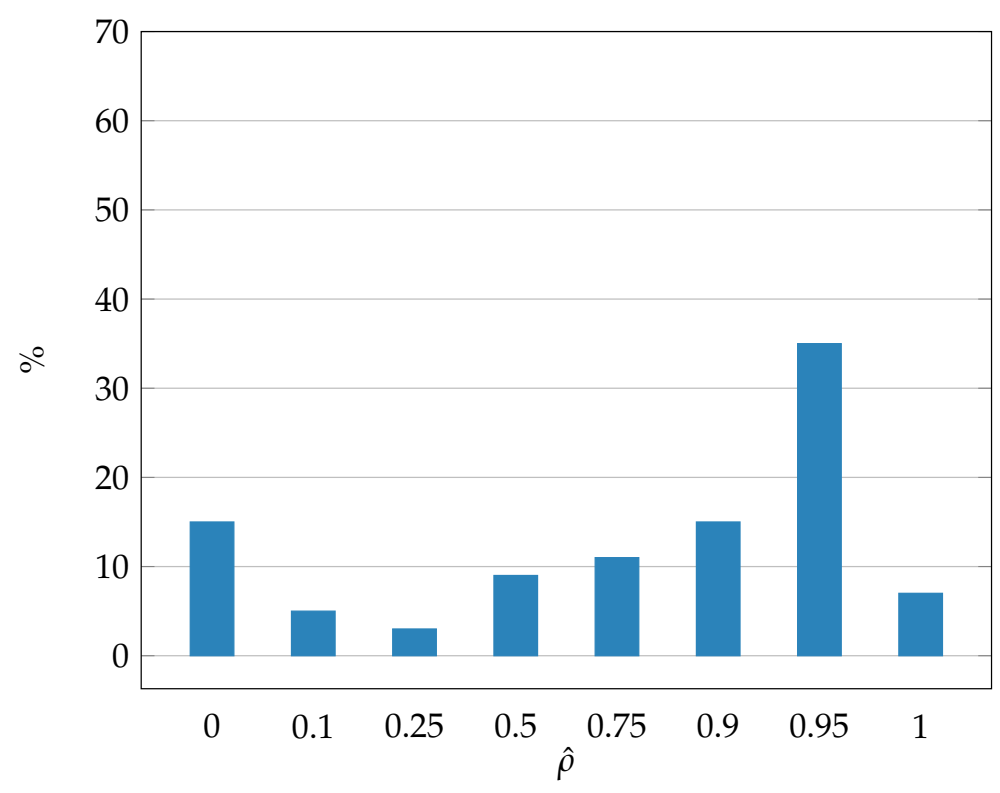

Notes: Beliefs were elicited using the infographic in Figure 6. 
Table 1: Intervention

Topic

Script

Partial

Full

Intervention Intervention

(P)

(F)

\begin{tabular}{|c|c|c|c|}
\hline $\begin{array}{l}\text { Initial beliefs about } \\
\text { private benefits of } \\
\text { ART }\end{array}$ & $\begin{array}{l}\text { Raise your hand if you believe that a person with } \\
\text { HIV can live a long and healthy life with ART. }\end{array}$ & $\mathrm{X}$ & \\
\hline $\begin{array}{l}\text { Private benefits of } \\
\text { ART }\end{array}$ & $\begin{array}{l}\text { A person who has HIV can live a long, healthy, } \\
\text { normal life, as long as he or she takes ART prop- } \\
\text { erly. }\end{array}$ & $\mathrm{X}$ & $X$ \\
\hline $\begin{array}{l}\text { ART mechanism: } \\
\text { reduction in viral } \\
\text { load }\end{array}$ & $\begin{array}{l}\text { If a person with HIV takes ART he will still have } \\
\text { HIV, but he will have a lower viral load, so the } \\
\text { symptoms may disappear. }\end{array}$ & $\mathrm{X}$ & $\mathrm{X}$ \\
\hline Infographic $(\mathrm{P})$ & Figure 3 & $\mathrm{X}$ & \\
\hline $\begin{array}{l}\text { Initial beliefs about } \\
\text { public benefit of } \\
\text { ART }\end{array}$ & $\begin{array}{l}\text { Imagine a couple. One person is HIV positive } \\
\text { and the other one is HIV negative. If the HIV- } \\
\text { positive person takes ART, does that reduce the } \\
\text { chance that the virus is passed to his or her part- } \\
\text { ner? Raise your hand if you think the answer is } \\
\text { yes. }\end{array}$ & & $\mathrm{X}$ \\
\hline $\begin{array}{l}\text { Public benefit of } \\
\text { ART }\end{array}$ & $\begin{array}{l}\text { Actually, it is true that if a person with HIV } \\
\text { takes ART, it can greatly reduce the chance of } \\
\text { spreading HIV. Imagine a certain area where no } \\
\text { one takes ART. In that area, } 100 \text { people got HIV } \\
\text { from their partners last year. If those partners } \\
\text { had been taking ART, only } 4 \text { people would have } \\
\text { gotten HIV. } 96 \text { of them would have remained } \\
\text { HIV negative. } \\
\text { If a person with HIV takes ART he will become } \\
96 \% \text { less contagious. This is true for both men } \\
\text { and women. This is because ART reduces the } \\
\text { amount of virus in the body. When there is very } \\
\text { little virus in the body, it is much less likely that } \\
\text { the virus will be transmitted from one person to } \\
\text { another. }\end{array}$ & & $\mathrm{X}$ \\
\hline Infographic $(\mathrm{F})$ & Figure 4 & & $X$ \\
\hline $\begin{array}{l}\text { Other information } \\
\text { about ART }\end{array}$ & $\begin{array}{l}\text { Only HIV-positive people should take ART. The } \\
\text { person who is taking ART must adhere properly, } \\
\text { taking the pills every day, exactly as instructed. } \\
\text { If he or she forgets to take the pills, the viral load } \\
\text { will go back up. }\end{array}$ & $\mathrm{X}$ & $\mathrm{X}$ \\
\hline $\begin{array}{l}\text { Other HIV } \\
\text { prevention methods }\end{array}$ & $\begin{array}{l}\text { For maximum protection from HIV, you should } \\
\text { be faithful to one partner and use condoms. }\end{array}$ & $\mathrm{X}$ & $X$ \\
\hline Availability of ART & Health clinics offer free HIV testing and ART. & $X$ & $X$ \\
\hline Questions & For other questions, ask at the health clinic. & $\mathrm{X}$ & $\mathrm{X}$ \\
\hline
\end{tabular}


Table 2: Balance on Village-Level Covariates

\begin{tabular}{|c|c|c|c|c|c|}
\hline & $\begin{array}{l}(1) \\
\text { Control }\end{array}$ & $\begin{array}{c}(2) \\
\text { Partial } \\
\text { intervention }\end{array}$ & $\begin{array}{c}\text { (3) } \\
\text { Full } \\
\text { intervention }\end{array}$ & $\begin{array}{c}(4) \\
\text { (1) vs. (2), } \\
\text { p-value }\end{array}$ & $\begin{array}{c}(5) \\
\text { (2) vs. (3) } \\
\text { p-value }\end{array}$ \\
\hline Distance to nearest health centre & 4.295 & 4.449 & 5.066 & 0.691 & 0.147 \\
\hline$\%$ of target ${ }^{\dagger}$ group tested for HIV pre-intervention & 1.479 & 1.315 & 0.885 & 0.593 & 0.126 \\
\hline$\%$ of target ${ }^{\dagger}$ group tested jointly pre-intervention & 0.357 & 0.345 & 0.163 & 0.927 & 0.103 \\
\hline$\%$ of target ${ }^{\dagger}$ group tested nearby pre-intervention & 0.702 & 0.817 & 0.454 & 0.519 & 0.056 \\
\hline$\%$ already taking ART pre-intervention & 2.495 & 2.546 & 2.080 & 0.881 & 0.177 \\
\hline \multicolumn{6}{|c|}{ Stratification variables } \\
\hline \multicolumn{6}{|l|}{$\begin{array}{l}\text { Nearest health facility } \\
\text { Village population }\end{array}$} \\
\hline Observations & 122 & 62 & 60 & & \\
\hline
\end{tabular}

Notes: $\mathrm{p}$-values are for a regression of the covariate on village intervention status. The pre-intervention period is 2.5 months. $\dagger$ Target population: age $15-49$, non-pregnant. The target population was calculated from the Malawian National Statistics Office census at the village level. 
Table 3: Beliefs Post-Intervention (4 Months): Does ART Reduce HIV Transmission?

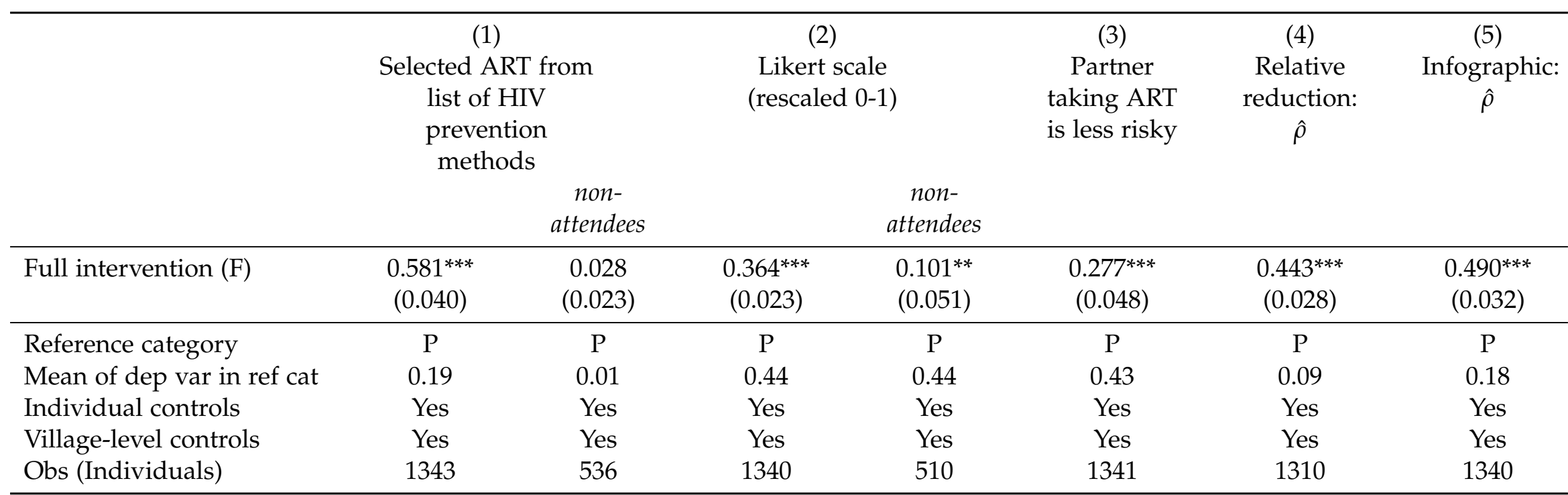

Notes: Data source is a survey to meeting attendees in partial $(\mathrm{P})$ and full $(\mathrm{F})$ intervention villages. Dependent variables are individual responses to survey questions. Reference category is partial intervention $(\mathrm{P}) . \rho=$ the relative reduction in HIV transmission associated with antiretroviral drugs. True value: $\rho=0.96$. (1) The outcome is an indicator for whether the respondent selected ART as one method of preventing the spread of HIV from the following list: faithfulness, abstinence, ART, circumcision, condoms, and mosquito nets. (2) Survey question: Do you agree or disagree with the following statement? If a person who is HIV positive takes ART it will reduce the chance that he transmits HIV to his or her partner. Likert scale: $5=$ strongly agree, $4=$ agree, $3=$ neither agree nor disagree, $2=$ disagree, $1=$ strongly disagree. The respondent's response was divided by 5 to obtain outcome variable. (3) The respondent used ten bottle caps to show their beliefs about the following probabilities. Absolute transmission probability: Ten couples are serodiscordant (one HIV positive and the other negative). Suppose they do not use condoms or ART drugs. After one year, how many will have transmitted HIV to their partner?Transmission probability with ART: Suppose instead that they are all taking ART. How many will transmit HIV to their partners? These two probabilities were used to calculate the relative reduction in risk associated with ART use; the true value is 0.96. (4) The outcome is an indicator for whether the respondent believes that a person on ART drugs is less likely to transmit HIV than a person who has never been tested for HIV. (5) The respondent's selection from Table 6 , converted into a measure of $\rho$ by subtracting from $100 \%$ and rescaling. The true value is 0.96 . The post-intervention period is approximately 4 months. All regressions are OLS, at the individual level, with individual-level controls, village-level controls and a constant. Individual-level controls: age, gender, married, employed, primary school educated, secondary school educated, has livestock, has a brick house. Village-level controls: variables listed in Table 2, village population and distance to each health facility. Robust standard errors are clustered at the village level and given in the parentheses with stars indicating *** $p<0.01, * * p<0.05, *$ $p<0.1$ 
Table 4: Attitudes and Beliefs about HIV Post-Intervention (4 Months )

\begin{tabular}{|c|c|c|c|c|c|}
\hline & \multicolumn{2}{|c|}{ Attitudes towards ART users } & \multicolumn{3}{|c|}{ Other beliefs about HIV } \\
\hline & $\begin{array}{c}(1) \\
=1 \text { if } \\
\text { respondent } \\
\text { prefers } \\
\text { untested } \\
\text { partner to } \\
\text { partner taking } \\
\text { ART }\end{array}$ & $\begin{array}{c}(2) \\
=1 \text { if } \\
\text { respondent } \\
\text { thinks a } \\
\text { person taking } \\
\text { ART won't } \\
\text { find a new } \\
\text { partner }\end{array}$ & $\begin{array}{l}(3) \\
\text { ART leads to } \\
\text { a long and } \\
\text { healthy life: } \\
\text { Likert scale } \\
\text { (rescaled 0-1) }\end{array}$ & $\begin{array}{l}(4) \\
\text { Absolute } \\
\text { transmission } \\
\text { probability } \\
\text { (one year) }\end{array}$ & $\begin{array}{c}\text { (5) } \\
\text { HIV } \\
\text { prevalence in } \\
\text { community }\end{array}$ \\
\hline Full intervention $(\mathrm{F})$ & $\begin{array}{c}-0.155^{* * *} \\
(0.034)\end{array}$ & $\begin{array}{c}-0.112^{* *} \\
(0.043)\end{array}$ & $\begin{array}{c}0.009 \\
(0.008)\end{array}$ & $\begin{array}{l}-0.011 \\
(0.009)\end{array}$ & $\begin{array}{c}0.013 \\
(0.013)\end{array}$ \\
\hline Reference category & $\mathrm{P}$ & $\mathrm{P}$ & $\mathrm{P}$ & $\mathrm{P}$ & $\mathrm{P}$ \\
\hline Mean of dep var in ref cat & 0.46 & 0.68 & 0.95 & 0.96 & 0.54 \\
\hline Individual controls & Yes & Yes & Yes & Yes & Yes \\
\hline Village-level controls & Yes & Yes & Yes & Yes & Yes \\
\hline Obs (Individuals) & 1224 & 1276 & 1340 & 1330 & 1224 \\
\hline
\end{tabular}

Notes: Data source is a survey to meeting attendees in partial $(\mathrm{P})$ and full $(\mathrm{F})$ intervention villages. Dependent variables are individual responses to survey questions. Reference category is partial intervention (P). (1) Respondent would prefer a partner who has never been tested for HIV to one who is taking ART drugs. (2) Respondent believes that a person taking ART will definitely not find a new sexual partner. (3) The respondent used ten bottle caps to show their beliefs about the one-year probability of HIV transmission for a serodiscordant couple who are not using condoms or taking ART. (4) The respondent used ten bottle caps to show their beliefs about the HIV prevalence in the village. (5) The average of four Likert-scale measures, rescaled to $(0,1)$ : current likelihood of having HIV, likelihood of getting HIV, level of worry about having HIV, level of worry about contracting HIV. The post-intervention period is approximately 4 months. All regressions are OLS, at the individual level, with individual-level controls, village-level controls and a constant. Individual-level controls: age, gender, married, employed, primary school educated, secondary school educated, has livestock, has a brick house. Village-level controls: variables listed in Table 2, village population and distance to each health facility. Robust standard errors are clustered at the village level and given in the parentheses with stars indicating ${ }^{* * *} p<0.01{ }^{* *} p<0.05,{ }^{*} p<0.1$ 
Table 5: HIV Tests Post-Intervention (4 Months)

(1)

$\%$ of population tested for HIV

Partial or full intervention (P or F) 0.153

Full intervention $(\mathrm{F}) \quad 0.830^{* *}$

\# study villages $<1 \mathrm{~km} \quad-0.026$

\# P or F villages $<1 \mathrm{~km} \quad-0.430$

\# F villages $<1 \mathrm{~km} \quad 0.942^{* *}$

(0.383)

Reference category

Control (C)

Mean of dep var in ref cat

3.09

Village-level controls

Yes

Proportional increase (F vs P) 27\%

Obs (Villages) 244

Notes: HIV testing rate is based on administrative data from 18 health facilities and survey. Dependent variable: \% of village target population tested for HIV post-intervention. Reference category is control (C). Target population: age 15-49, non-pregnant. The target population was calculated from the Malawian National Statistics Office census at the village level. The post-intervention period is 4 months. All regressions are at the village level, with village-level controls and include a constant. Village-level controls: variables listed in Table 2, village population and distance to each health facility. Robust standard errors are given in the parentheses with stars indicating *** $p<0.01$, ${ }^{* *} p<0.05{ }^{*} p<0.1$. 
Table 6: HIV Testing Location

\begin{tabular}{|c|c|c|c|c|}
\hline & \multicolumn{4}{|c|}{$\%$ of population tested for HIV } \\
\hline & $(1)$ & $(2)$ & (3) & (4) \\
\hline & Nearby & Far & Nearby & Far \\
\hline Full intervention $(\mathrm{F})$ & $\begin{array}{c}0.322 \\
(0.328)\end{array}$ & $\begin{array}{l}0.336 \\
(0.294)\end{array}$ & & \\
\hline Proportion of village with high beliefs $(\hat{\rho} \geq 0.95)$ & & & $\begin{array}{l}1.076^{*} \\
(0.593)\end{array}$ & $\begin{array}{l}-0.686 \\
(0.654)\end{array}$ \\
\hline Proportion of village with moderate beliefs $(0<\hat{\rho}<0.95)$ & & & $\begin{array}{l}-0.130 \\
(0.604)\end{array}$ & $\begin{array}{c}0.391 \\
(0.534)\end{array}$ \\
\hline Reference category & Partial (P) & Partial (P) & & \\
\hline Mean of dep var in ref cat & 1.65 & 1.43 & & \\
\hline Village-level controls & Yes & Yes & Yes & Yes \\
\hline Obs (Villages) & 122 & 122 & 119 & 119 \\
\hline
\end{tabular}

Notes: HIV testing rate and location are based on administrative data from 18 health facilities. Dependent variable: \% of village target population tested for HIV post-intervention far/nearby. Far clinics defined as $>2 \mathrm{~km}$ further than nearest free clinic. (1)-(2): Reference category is partial intervention (P). (4)-(6): $\rho=$ the relative reduction in HIV transmission associated with antiretroviral drugs. True value: $\rho=0.96$. Community beliefs about $\rho$ are approximated by the village-level average of beliefs, as obtained from the survey using the infographic in Figure 6. Omitted reference category: proportion of village with low beliefs $\rho=0$. Target population: age $15-49$, non-pregnant. The target population was calculated from the Malawian National Statistics Office census at the village level. The post-intervention period is 4 months. All regressions are at the village level, with village-level controls and include a constant. Village-level controls: variables listed in Table 2, village population and distance to each health facility. Robust standard errors are given in the parentheses with stars indicating ${ }^{* * *} p<0.01,{ }^{* *} p<0.05,{ }^{*} p<0.1$. 
Table 7: Common Knowledge of Public Benefit of ART and HIV Testing

\begin{tabular}{|c|c|c|c|c|}
\hline & \multicolumn{3}{|c|}{ HIV test: post-intervention } & \multirow{2}{*}{$\frac{\text { pre-intervention }}{(4)}$} \\
\hline & $(1)$ & (2) & (3) & \\
\hline Respondent believes $\rho \geq 0.95$ & & $\begin{array}{c}-0.033 \\
(0.048)\end{array}$ & $\begin{array}{c}-0.005 \\
(0.072)\end{array}$ & $\begin{array}{c}0.012 \\
(0.060)\end{array}$ \\
\hline Respondent thinks spouse believes $\rho \geq 0.95$ & & & $\begin{array}{c}-0.073 \\
(0.084)\end{array}$ & $\begin{array}{c}-0.026 \\
(0.069)\end{array}$ \\
\hline Respondent thinks community believes $\rho \geq 0.95$ & $\begin{array}{l}0.083^{*} \\
(0.043)\end{array}$ & $\begin{array}{l}0.106^{\text {** }} \\
(0.050)\end{array}$ & $\begin{array}{l}0.135^{* *} \\
(0.055)\end{array}$ & $\begin{array}{l}-0.055 \\
(0.054)\end{array}$ \\
\hline Village fixed effects & Yes & Yes & Yes & Yes \\
\hline Individual controls & Yes & Yes & Yes & Yes \\
\hline Obs (Individuals) & 1333 & 1330 & 1079 & 1079 \\
\hline
\end{tabular}

Notes: Data source is a survey to meeting attendees in partial (P) and full (F) intervention villages. Dependent variable: indicator for whether the respondent reported having an HIV test. $\rho=$ the relative reduction in HIV transmission associated with antiretroviral drugs. True value: $\rho=0.96$. Beliefs about $\rho$ are obtained from the survey using the infographic in Figure 6. (5)-(8): sample restricted to married respondents. The pre-intervention period is 5 months. The post-intervention period is approximately 4 months. All regressions are OLS, at the individual level, with individual-level controls, village fixed effects. Individual-level controls: age, gender married, employed, primary school educated, secondary school educated, has livestock, has a brick house. Robust standard errors are clustered at the village level and given in the parentheses with stars indicating ${ }^{* * *} p<0.01,{ }^{* *} p<0.05,{ }^{*} p<0.1$ 
Table 8: Antiretroviral Therapy (ART) Initiation Post-Intervention (4 Months)

(1)

$\%$ of population initiated ART

Partial or full intervention (P or F) $0.198^{* *}$

Full intervention $(\mathrm{F})$

\# study villages $<1 \mathrm{~km}$

\# $\mathrm{P}$ or F villages $<1 \mathrm{~km}$

\# F villages $<1 \mathrm{~km}$

0.076

$(0.076)$

Reference category

Mean of dep var in ref cat

Control (C)

Village-level controls

.17

Proportional increase $(\mathrm{P}+\mathrm{F}$ vs $\mathrm{C})$

Yes

Obs (Villages)

Notes: ART initiation rate is based on administrative data from 18 health facilities and survey. Dependent variable: \% of village target population initiated ART post-intervention. Reference category is control (C). Target population: age 15-49, non-pregnant. The target population was calculated from the Malawian National Statistics Office census at the village level. The post-intervention period is 4 months. All regressions are at the village level, with village-level controls and include a constant. Village-level controls: variables listed in Table 2, village population and distance to each health facility. Robust standard errors are given in the parentheses with stars indicating ${ }^{* * *} p<0.01,{ }^{* *} p<0.05{ }^{*} p<0.1$. 
Table 9: Risk Compensation

\begin{tabular}{lcc}
\hline & $(1)$ & $(2)$ \\
& Sex acts & Condoms used \\
\hline Full intervention (F) & 0.462 & -0.054 \\
& $(0.302)$ & $(0.115)$ \\
\hline Reference category & $\mathrm{P}$ & $\mathrm{P}$ \\
Mean of dep var in ref cat & 1.59 & 0.34 \\
Individual controls & Yes & Yes \\
Village-level controls & Yes & Yes \\
Obs (Individuals) & 1330 & 1325 \\
\hline
\end{tabular}

Notes: Data source is a survey to meeting attendees in partial $(\mathrm{P})$ and full $(\mathrm{F})$ intervention villages. Dependent variables are individual responses to survey questions. Reference category is partial intervention (P). (1) Number of sex acts, recalled over past 7 days. (2) Number of condoms used, recalled over past 7 days. $\rho=$ the relative reduction in HIV transmission associated with antiretroviral drugs. True value: $\rho=0.96$. Beliefs about $\rho$ are obtained from the survey using the infographic in Figure 6 . The post-intervention period is approximately 4 months. All regressions are OLS, at the individual level, with individual-level controls, village-level controls and a constant. Individual-level controls: age, gender, married, employed, primary school educated, secondary school educated, has livestock, has a brick house. Village-level controls: variables listed in Table 2, village population and distance to each health facility. Robust standard errors are clustered at the village level and given in the parentheses with stars indicating *** $p<0.01,{ }^{* *} p<0.05, * p<0.1$ 
Table 10: Pressure to Test for HIV

\begin{tabular}{|c|c|c|c|c|}
\hline & \multirow{2}{*}{$\begin{array}{c}\text { Joint tests } \\
(1) \\
\% \text { of } \\
\text { population } \\
\text { joint tested for } \\
\text { HIV }\end{array}$} & \multicolumn{3}{|c|}{ Survey measures } \\
\hline & & $\begin{array}{c}(2) \\
=1 \text { if } \\
\text { respondent } \\
\text { tested for HIV } \\
\text { due to } \\
\text { pressure }\end{array}$ & $\begin{array}{l}\quad(3) \\
=1 \text { if } \\
\text { respondent } \\
\text { would prefer } \\
\text { joint testing to } \\
\text { private testing }\end{array}$ & $\begin{array}{c}(4) \\
\text { WTP for joint } \\
\text { testing over } \\
\text { private testing }\end{array}$ \\
\hline Partial or full intervention (P or F) & $\begin{array}{l}-0.089 \\
(0.181)\end{array}$ & & & \\
\hline Full intervention $(\mathrm{F})$ & $\begin{array}{c}0.023 \\
(0.164)\end{array}$ & $\begin{array}{c}0.006 \\
(0.007)\end{array}$ & $\begin{array}{l}-0.017 \\
(0.017)\end{array}$ & $\begin{array}{c}-6.119 \\
(15.704)\end{array}$ \\
\hline \# study villages $<1 \mathrm{~km}$ & $\begin{array}{l}-0.015 \\
(0.104)\end{array}$ & & & \\
\hline$\# \mathrm{P}$ or $\mathrm{F}$ villages $<1 \mathrm{~km}$ & $\begin{array}{c}-0.041 \\
(0.132)\end{array}$ & & & \\
\hline$\# \mathrm{~F}$ villages $<1 \mathrm{~km}$ & $\begin{array}{c}0.047 \\
(0.185)\end{array}$ & & & \\
\hline Reference category & $\mathrm{C}$ & $\mathrm{P}$ & $\mathrm{P}$ & $\mathrm{P}$ \\
\hline Mean of dep var in ref cat & .44 & 0.01 & 0.94 & 105.40 \\
\hline Individual controls & No & Yes & Yes & Yes \\
\hline Village-level controls & Yes & Yes & Yes & Yes \\
\hline Obs (Villages) & 244 & & & \\
\hline Obs (Individuals) & & 1010 & 1010 & 1006 \\
\hline
\end{tabular}

Notes: (1) Data source: administrative data from 18 health facilities in Zomba District. Dependent variable: \% of village target population joint tested for HIV postintervention. Target population: age 15-49, non-pregnant. The target population was calculated from the Malawian National Statistics Office census at the village level. The post-intervention period is 4 months. Regression is OLS at the village level, with village-level controls and a constant. Reference category is control (C). (2)-(4): Data source: survey to meeting attendees in intervention villages. Dependent variables are individual responses to survey questions. Sample restricted to married respondents. The post-intervention period is approximately 4 months. All regressions are OLS, at the individual level, and with individual-level controls, village-level controls and a constant. Reference category is partial intervention (P). Individual-level controls: age, gender, employed, primary school educated, secondary school educated, has livestock, has a brick house. (2) Respondent sought HIV test due to pressure. (3) Respondent would prefer a joint test over private test. Sample restricted to married respondents. (4) Respondent's willingness to pay for door-to-door joint testing campaign vs. private testing (Malawi Kwacha: 400MK = 1USD). Village-level controls: Table 2, village population and distance to each health facility. Robust standard errors are given in the parentheses with stars indicating $* * * ~ p<0.01$, $* *$ $p<0.05,{ }^{*} p<0.1$. 


\section{Appendices}

\section{A Equilibria}

In this section, we characterize the pure-strategy Perfect Bayesian Equilibria of the model presented in Section 3. The partially separating equilibrium, in which a fraction $S$ of the population rejects potential sexual partners who have been tested, was analyzed in Section 3.2. Proposition 1 claims that there is only one other type of equilibrium: a pooling equilibrium.

\section{A.1 The Pooling Equilibrium}

For some parameter values, the game described in Section 3 has a class of pure-strategy Perfect Bayesian Equilibria in which a fraction $P$ of the population $\mathcal{B}$ consent only if their match has been tested, and the remaining fraction $1-P$ consent to all matches. $P$ must be large enough to induce universal testing, because the equilibrium relies on extreme off-equilibrium beliefs about those who do not test for HIV. In this sense, the pooling equilibrium is not stable (it does not satisfy the D1 criterion). Intuitively, highrisk individuals have more to gain from testing, so it is not natural to believe that a person who has not been tested is more risky than someone who has. Nonetheless, this equilibrium satisfies the definition of a Perfect Bayesian Equilibrium.

Fix $P$, and consider the strategy of each $a \in \mathcal{A}$. He will not test far away, as it is costly, and there is no benefit to hiding the decision since all matches consent to those who test. His benefit to testing nearby is access to ART drugs plus the guarantee that his potential sexual partner will consent, $\theta_{a} v+P y_{a}$, and the cost is $c^{n}$. He will therefore test nearby if

$$
\theta_{a} v+P y_{a}>c^{n}
$$

High-risk types $\theta_{a}=\theta_{H}$ will always test, because we assumed $\theta_{H} v>c^{n}$.

The proportion of low-risk types who test is equal to

$$
\mathbb{P}\left(\theta_{L} v+P y_{a}>c^{n}\right) .
$$

If this measure is less than one, that is, testing is not universal, then this is not an equilibrium. To see why, consider the beliefs of $b \in \mathcal{B}$. If her match has not been tested, she concludes that his type is $\theta=\theta_{L}$. But in this case, she will consent, because we assumed that everyone consents to a person of average risk (assumption (6)), and therefore $P=0$. This implies that for parameters such that

$$
\mathbb{P}\left(\theta_{L} v+y_{a}>c^{n}\right)<1
$$

there is no pooling equilibrium, because even if $P=1$, that is, everyone rejects those who have not been tested, universal testing will not be achieved. Some low-risk types are not sufficiently motivated by the prospect of a sexual relationship to bother seeking an HIV test. 
Conversely, a pooling equilibrium exists for any $0<P \leq 1$ such that

$$
\mathbb{P}\left(\theta_{L} v+P y_{a}>c^{n}\right)=1
$$

All agents $a \in \mathcal{A}$ best respond to the strategies of agents $\mathcal{B}$ by testing nearby. In this case, beliefs about a match who has not been tested $\left(\sigma_{a}=0\right)$ can take any value, as this action is off the equilibrium path. In order to demonstrate the existence of the equilibrium, we set these beliefs equal to $\hat{\theta}_{b}(0)=1$ for all $b \in[0, P]$ and $\hat{\theta}_{b}(0)=0$ for all $b \in(P, 1]$. That is, a fraction $P$ of the population believe that a person who has not been tested is HIV positive with probability one. The remaining fraction $1-P$ believe that a person who has not been tested is HIV negative. In this case, because it is never worth consenting to a match who is infected and untreated, $\mathbb{P}\left(y_{b}<c^{h} \tau+c^{o}\right)=1$ (assumption (7)), all $b \in[0, P]$ will reject a partner who has not been tested. Meanwhile, because we have assumed it is always worth consenting to an average-risk match (assumption (6)),

$$
\mathbb{P}\left(y_{b}>0\right) \geq \mathbb{P}\left(y_{b}>\bar{h}\left(c^{h} \tau+c^{o}\right)\right)=1,
$$

all $b \in(P, 1]$ will consent to any match. In equilibrium, HIV testing is universal.

\section{A.2 Ruling out Other Equilibria}

We have described two classes of equilibria. A partially separating equilibrium is one in which a fraction $S \in[0,1]$ of the population rejects matches who have been tested for $\mathrm{HIV}$, and otherwise all matches obtain consent ${ }^{41}$. A pooling equilibrium is one in which a fraction $P \in(0,1]$ of the population rejects those who have not been tested, and otherwise all matches obtain consent. We now describe and rule out other potential types of equilibria.

\section{There is no equilibrium in which any agent rejects all matches}

In the case of either universal testing or universal abstention from testing, consistent beliefs on the equilibrium path will equal the average HIV prevalence in the population: $\hat{\theta}_{b}=\bar{h}$, and by assumption (6), $b$ will consent, because

$$
y_{b}>\bar{h}\left(c^{h} \tau+c^{o}\right)>\bar{h}\left(c^{h} \tau\left(1-\hat{\rho}_{b}\right)+c^{o}\right)
$$

Consider the case in which some agents test and others do not. Because of random matching, all $a \in \mathcal{A}$ face the same expected cost of seeking a test. However, high-risk types have more to gain. This implies adverse selection among those who test, and those who do not test are therefore lower-risk than the average member of the population. So, consistent beliefs about those who do not test are $\hat{\theta}_{b}(0)<\bar{h}$, and by assumption (6), $b$ will consent to those who do not seek an HIV test.

\footnotetext{
${ }^{41}$ We are including the case $S=0$, in which all $b \in \mathcal{B}$ consent to any match, under the title partially separating. Within this equilbrium, $S=0$ describes the case in which the cost of discrimination is zero.
} 
There is no equilibrium in which different agents adopt opposite strategies towards matches who have been tested

We can now restrict attention to equilibria in which each agent $b \in \mathcal{B}$ adopts a strategy of consenting to some or all matches. The partially separating and pooling equilibria cover the cases in which some agents always consent, and those who do not always consent all adopt the same strategies.

The only other potential class of equilibrium is one in which different agents $b \in \mathcal{B}$ adopt opposite strategies towards those who seek an HIV test. That is, $S>0$ and $P>0$.

Suppose $S>P>0$. In this case, all low-risk types will not test, and some high-risk types may not either. Among those who do not test, the average risk is less than that the average risk in the population, so consistent beliefs are $\hat{\theta}_{b}(0)<\bar{h}$. By assumption (6), all $b \in \mathcal{B}$ will consent to those who do not test, so $P=0$, which is a contradiction.

Suppose instead that $P>S>0$. In this case, all high-risk types will test, as the net cost of discrimination is negative $(S-P<0)$. We consider two subcases. First, suppose this does not induce universal testing. That is,

$$
\mathbb{P}\left(\theta_{L} v+(P-S) y_{a}>c^{n}\right)<1 .
$$

Then any person who does not test is a low-risk type, so rational beliefs are $\hat{\theta}_{b}(0)=\theta_{L}$. By assumption (6), all $b \in \mathcal{B}$ will consent to those who do not test, so $P=0$, which again forms a contradiction. Instead, suppose that this does induce universal testing, so

$$
\mathbb{P}\left(\theta_{L} v+(P-S) y_{a}>c\right)=1 .
$$

Now, rational beliefs about those who do seek an HIV test are $\hat{\theta}_{b}(1)=\bar{h}$, so by assumption (6) everyone will consent. This implies $S=0$.

\section{B Alternative Definitions of Nearby and Far Away Testing}

Throughout the paper, we have defined a nearby test as an HIV test that takes place at most two kilometers further than the nearest clinic to a client's home village. In this section we reproduce results on the choice of testing location using different buffer zones around the nearest clinic.

Results with no buffer zone are shown in Table A1. In these tables, we say an HIV test took place nearby only if it took place at the nearest health facility to the village, as measured by GPS coordinates. Using this definition, we see no significant link between beliefs about the public benefit of ART and the number of nearby tests. This definition of nearby is unsatisfactory as it does not take into account the fact that for some villages, the nearest clinic as measured in travel time may not be the same as the nearest clinic by GPS. Indeed, $61 \%$ of villages do not have a single HIV test taking place at the nearest clinic during the pre- and post-intervention periods combined. In addition, if a clinic is less than a kilometer further than the nearest clinic, we should probably not categorize that clinic visit as far away and claim that the choice is due to stigma. On this margin, other factors such as wait time or quality may play a larger role.

For the above reasons, a good definition of nearby should include a buffer zone. Table A2 shows results with a buffer zone of one kilometer, that is, a visit is considered nearby if it takes place at most one kilometer further than the nearest clinic. Table A3 
shows results with a buffer zone of three kilometers. These choices both provide results that are quantitatively similar to those presented in the main specifications with a buffer zone of two kilometers (Table 6). 
Table A1: HIV Testing Location (0km Buffer)

\begin{tabular}{|c|c|c|c|c|}
\hline & \multicolumn{4}{|c|}{$\%$ of population tested for HIV } \\
\hline & (1) & $(2)$ & (3) & $(4)$ \\
\hline & Nearby & Far & Nearby & Far \\
\hline Full intervention $(F)$ & $\begin{array}{c}0.032 \\
(0.250)\end{array}$ & $\begin{array}{c}0.586 \\
(0.357)\end{array}$ & & \\
\hline Proportion of village with high beliefs $(\hat{\rho} \geq 0.95)$ & & & $\begin{array}{c}0.408 \\
(0.402)\end{array}$ & $\begin{array}{c}-0.108 \\
(0.782)\end{array}$ \\
\hline Proportion of village with moderate beliefs $(0<\hat{\rho}<0.95)$ & & & $\begin{array}{l}-0.225 \\
(0.458)\end{array}$ & $\begin{array}{c}0.406 \\
(0.636)\end{array}$ \\
\hline Reference category & Partial (P) & Partial (P) & & \\
\hline Mean of dep var in ref cat & 1.06 & 2.03 & & \\
\hline Village-level controls & Yes & Yes & Yes & Yes \\
\hline Obs (Villages) & 122 & 122 & 119 & 119 \\
\hline
\end{tabular}

Notes: HIV testing rate and location are based on administrative data from 18 health facilities. Dependent variable: \% of village target population tested for HIV post-intervention far/nearby. Far clinics defined as $>0 \mathrm{~km}$ further than nearest free clinic. (1)-(2): Reference category is partial intervention (P). (4)-(6): $\rho=$ the relative reduction in HIV transmission associated with antiretroviral drugs. True value: $\rho=0.96$. Community beliefs about $\rho$ are approximated by the village-level average of beliefs, as obtained from the survey using the infographic in Figure 6. Omitted reference category: proportion of village with low beliefs $\rho=0$. Target population: age $15-49$, non-pregnant. The target population was calculated from the Malawian National Statistics Office census at the village level. The post-intervention period is 4 months. All regressions are at the village level, with village-level controls and include a constant. Village-level controls: variables listed in Table 2, village population and distance to each health facility. Robust standard errors are given in the parentheses with stars indicating ${ }^{* * *} p<0.01{ }^{* *} p<0.05,^{*} p<0.1$. 
Table A2: HIV Testing Location (1km Buffer)

\begin{tabular}{|c|c|c|c|c|}
\hline & \multicolumn{4}{|c|}{$\%$ of population tested for HIV } \\
\hline & (1) & $(2)$ & (3) & $(4)$ \\
\hline & Nearby & Far & Nearby & Far \\
\hline Full intervention $(F)$ & $\begin{array}{c}0.287 \\
(0.312)\end{array}$ & $\begin{array}{c}0.289 \\
(0.315)\end{array}$ & & \\
\hline Proportion of village with high beliefs $(\hat{\rho} \geq 0.95)$ & & & $\begin{array}{c}0.918 \\
(0.560)\end{array}$ & $\begin{array}{c}-0.691 \\
(0.702)\end{array}$ \\
\hline Proportion of village with moderate beliefs $(0<\hat{\rho}<0.95)$ & & & $\begin{array}{c}0.032 \\
(0.602)\end{array}$ & $\begin{array}{c}0.081 \\
(0.565)\end{array}$ \\
\hline Reference category & Partial (P) & Partial (P) & & \\
\hline Mean of dep var in ref cat & 1.3 & 1.79 & & \\
\hline Village-level controls & Yes & Yes & Yes & Yes \\
\hline Obs (Villages) & 122 & 122 & 119 & 119 \\
\hline
\end{tabular}

Notes: HIV testing rate and location are based on administrative data from 18 health facilities. Dependent variable: \% of village target population tested for HIV post-intervention far/nearby. Far clinics defined as $>1 \mathrm{~km}$ further than nearest free clinic. (1)-(2): Reference category is partial intervention (P). (4)-(6): $\rho=$ the relative reduction in HIV transmission associated with antiretroviral drugs. True value: $\rho=0.96$. Community beliefs about $\rho$ are approximated by the village-level average of beliefs, as obtained from the survey using the infographic in Figure 6. Omitted reference category: proportion of village with low beliefs $\rho=0$. Target population: age $15-49$, non-pregnant. The target population was calculated from the Malawian National Statistics Office census at the village level. The post-intervention period is 4 months. All regressions are at the village level, with village-level controls and include a constant. Village-level controls: variables listed in Table 2, village population and distance to each health facility. Robust standard errors are given in the parentheses with stars indicating ${ }^{* * *} p<0.01{ }^{* *} p<0.05,^{*} p<0.1$. 
Table A3: HIV Testing Location (3km Buffer)

\begin{tabular}{|c|c|c|c|c|}
\hline & \multicolumn{4}{|c|}{$\%$ of population tested for HIV } \\
\hline & (1) & $(2)$ & (3) & $(4)$ \\
\hline & Nearby & Far & Nearby & Far \\
\hline Full intervention $(F)$ & $\begin{array}{c}0.358 \\
(0.327)\end{array}$ & $\begin{array}{c}0.339 \\
(0.297)\end{array}$ & & \\
\hline Proportion of village with high beliefs $(\hat{\rho} \geq 0.95)$ & & & $\begin{array}{l}1.187^{* *} \\
(0.593)\end{array}$ & $\begin{array}{c}-0.712 \\
(0.621)\end{array}$ \\
\hline Proportion of village with moderate beliefs $(0<\hat{\rho}<0.95)$ & & & $\begin{array}{c}0.009 \\
(0.635)\end{array}$ & $\begin{array}{c}0.267 \\
(0.462)\end{array}$ \\
\hline Reference category & Partial (P) & Partial (P) & & \\
\hline Mean of dep var in ref cat & 1.92 & 1.16 & & \\
\hline Village-level controls & Yes & Yes & Yes & Yes \\
\hline Obs (Villages) & 122 & 122 & 119 & 119 \\
\hline
\end{tabular}

Notes: HIV testing rate and location are based on administrative data from 18 health facilities. Dependent variable: \% of village target population tested for HIV post-intervention far/nearby. Far clinics defined as $>3 \mathrm{~km}$ further than nearest free clinic. (1)-(2): Reference category is partial intervention $(\mathrm{P})$. (4)-(6): $\rho=$ the relative reduction in HIV transmission associated with antiretroviral drugs. True value: $\rho=0.96$. Community beliefs about $\rho$ are approximated by the village-level average of beliefs, as obtained from the survey using the infographic in Figure 6. Omitted reference category: proportion of village with low beliefs $\rho=0$. Target population: age $15-49$, non-pregnant. The target population was calculated from the Malawian National Statistics Office census at the village level. The post-intervention period is 4 months. All regressions are at the village level, with village-level controls and include a constant. Village-level controls: variables listed in Table 2, village population and distance to each health facility. Robust standard errors are given in the parentheses with stars indicating ${ }^{* * *} p<0.01,{ }^{* *} p<0.05,{ }^{*} p<0.1$. 